\title{
Ultrawideband Noise Radar Imaging of Impenetrable Cylindrical Objects Using Diffraction Tomography
}

\author{
Hee Jung Shin, ${ }^{1}$ Ram M. Narayanan, ${ }^{1}$ and Muralidhar Rangaswamy ${ }^{2}$ \\ ${ }^{1}$ The Pennsylvania State University, University Park, PA 16802, USA \\ ${ }^{2}$ Air Force Research Laboratory, Wright-Patterson Air Force Base, OH 45433, USA \\ Correspondence should be addressed to Ram M. Narayanan; ram@ee.psu.edu
}

Received 31 July 2014; Revised 18 November 2014; Accepted 26 November 2014; Published 24 December 2014

Academic Editor: Gian Luigi Gragnani

Copyright (c) 2014 Hee Jung Shin et al. This is an open access article distributed under the Creative Commons Attribution License, which permits unrestricted use, distribution, and reproduction in any medium, provided the original work is properly cited.

\begin{abstract}
Ultrawideband (UWB) waveforms achieve excellent spatial resolution for better characterization of targets in tomographic imaging applications compared to narrowband waveforms. In this paper, two-dimensional tomographic images of multiple scattering objects are successfully obtained using the diffraction tomography approach by transmitting multiple independent and identically distributed (iid) UWB random noise waveforms. The feasibility of using a random noise waveform for tomography is investigated by formulating a white Gaussian noise (WGN) model using spectral estimation. The analytical formulation of object image formation using random noise waveforms is established based on the backward scattering, and several numerical diffraction tomography simulations are performed in the spatial frequency domain to validate the analytical results by reconstructing the tomographic images of scattering objects. The final image of the object based on multiple transmitted noise waveforms is reconstructed by averaging individually formed images which compares very well with the image created using the traditional Gaussian pulse. Pixel difference-based measure is used to analyze and estimate the image quality of the final reconstructed tomographic image under various signal-to-noise ratio (SNR) conditions. Also, preliminary experiment setup and measurement results are presented to assess the validation of simulation results.
\end{abstract}

\section{Introduction}

Research on the use of random or pseudorandom noise transmit signals in radar has been conducted since the 1950s $[1,2]$. Noise radar has been considered a promising technique for the covert identification of target objects due to several advantages, such as excellent electronic countermeasure (ECM), low probability of detection (LPD), low probability of interception (LPI) features, and relatively simple hardware architectures [3-5]. Also, advances in signal and imaging processing techniques in radar systems have progressed so that multidimensional representations of the target object can be obtained [6].

In general, radar imaging tends to be formulated in the time domain to exploit efficient back-projection algorithms, generate accurate shape features of the target object, and provide location data [7]. For multistatic radar systems, the images of a target are reconstructed based on range profiles obtained from the distributed sensor elements. When a transmitter radiates a waveform, spatially distributed receivers collect samples of the scattered field which are related to the electrical parameters of the target object. For the next iteration, a different transmitter is activated, and the scattered field collection process is repeated. Finally, all collected scattered field data are relayed for signal processing and subsequent image formation algorithms.

Tomography-based radar imaging algorithms have been developed based on microwave image reconstruction method [9], characterizing the material property profiles of the target object in the frequency domain and reconstructing specific scattering features inside the interrogation medium by solving the inverse scattering problem. The capability of microwave imaging techniques has been found attractive in malignant breast cancer detection [10-13], civil infrastructure assessment [14-16], and homeland security [1719] applications due to the advantages of nondestructive 
diagnosis and evaluation of obscured objects. The quality of the reconstructed image for different values of the electrical contrast for a UWB imaging system was investigated and published for both low-contrast and high-contrast object cases. For low-contrast objects, the obtained target image using a single frequency achieves a good reconstruction of the electrical contrast that is almost equivalent to the one obtained with the entire UWB frequency range. For the highcontrast case, while the formation of a Moiré pattern affects the single frequency reconstruction, this artifact does not appear in the UWB frequency image [20]. Thus, UWB radar tomography is expected to provide advantages over the single or narrow band frequency operation in terms of resolution and accuracy for any target object.

The goal of this paper is to demonstrate successful image reconstruction of the cylindrical conducting objects using the diffraction tomography theorem for bistatic UWB noise radar systems. The paper is organized as follows. First, the paper defines the characteristics of UWB random noise signal and discusses the shortcomings of using such noise signal as a radar transmit waveform in tomographic image reconstruction process in Section 2. The empirical solution to bypass the shortcoming of using UWB random noise waveform is also proposed. The formulations of the image reconstruction of two-dimensional scattering geometry of a bistatic imaging radar system using Fourier diffraction theorem under the assumption of plane wave illumination are presented in Section 3. In Section 4, the numerical simulation results of diffraction tomography using UWB random noise waveforms show that the tomographic image of the target is successfully reconstructed. The image quality measures of the reconstructed images, SNR effects for multiple transmissions of UWB random noise waveforms, and preliminary experimental validation are discussed in Section 5. Conclusions are presented in Section 6.

\section{Analysis of White Gaussian Noise Model}

The main advantage of transmitting a random noise waveform is to covertly detect and image a target without alerting others about the presence of radar system. Such LPI characteristics of the noise radar are guaranteed because the transmitted random noise waveform is constantly varying and never repeats itself exactly [21]. The random noise waveform can be experimentally generated simply by amplifying the thermal noise generated in resistors or noise diodes while maintaining relatively flat spectral density versus frequency [22]. Hence, relatively simple hardware designs can be achieved for noise radars compared to the conventional radar systems using complicated signal modulation schemes.

For a random noise waveform model, let $x[n]$ be a discrete time WSS and ergodic random process and a sequence of iid random variable drawn from a Gaussian distribution, $\mathcal{N}\left(0, \sigma^{2}\right) . x[n]$ defined herein is white Gaussian noise; that is, its probability density function follows a Gaussian distribution and its power spectral density is ideally a nonzero constant for all frequencies. However, the finite number of random noise amplitude samples must be chosen for waveform generation for any numerical simulations and practical experiments.

Assume that a sequence of only $l$ samples of $x[n]$ is selected for generating a white Gaussian noise. In this case, the estimate for the power spectral density, $\widehat{S}_{l}(\omega)$, is given by [23]

$$
I_{l}(\omega)=\widehat{S}_{l}(\omega)=\sum_{m=-(l-1)}^{l-1} \widehat{r}_{l}[m] e^{-j \omega m}
$$

where $\widehat{r}_{l}[m]$ is the estimate for the autocorrelation sequence. $I_{l}(\omega)$ is defined as the periodogram estimate, and the rigorous analysis of the expected value and variance of the periodogram estimate for any arbitrary $\omega$ is described in [23-25]. The expected value of the periodogram estimate is $[23,24]$

$$
\begin{aligned}
\mathrm{E}\left[I_{l}(\omega)\right] & =\sum_{m=-(l-1)}^{l-1} \mathrm{E}\left[\widehat{r}_{l}[m]\right] e^{-j \omega m} \\
& =\sum_{m=-(l-1)}^{l-1}\left(1-\frac{|m|}{l}\right) r_{l}[m] e^{-j \omega m},
\end{aligned}
$$

which suggests that $I_{l}(\omega)$ is a biased estimator. However, it is considered to be asymptotically unbiased as $l$ approaches infinity. In this case, the expected value of $I_{l}(\omega)$ becomes a constant such that

$$
\mathrm{E}\left[I_{l}(\omega)\right] \simeq S_{x}(\omega)=\sigma_{x}^{2} \quad \text { as } l \longrightarrow \infty
$$

The variance of the periodogram estimate of the white Gaussian noise waveform formed by a sequence of $l$ samples is given by $[23,24]$

$$
\operatorname{VAR}\left[I_{l}(\omega)\right]=S_{x}(\omega)^{2}\left(1+\left(\frac{\sin (\omega l)}{l \sin (\omega)}\right)^{2}\right)
$$

which is proportional to the square of the power spectrum density and does not approach zero as $l$ increases. In order to decrease the variance of $I_{l}(\omega)$, the periodogram averaging method has been proposed by Bartlett [26]. The average of $K$ independent and identically distributed periodograms on samples of size $l$ is given by

$$
\bar{I}_{l, K}(\omega)=\frac{1}{K} \sum_{i=0}^{K-1} I_{l, i}(\omega),
$$

and the expected value of the average with $K$ iid periodogram estimate is written as [23]

$$
\begin{aligned}
\mathrm{E}\left[\bar{I}_{l, K}(\omega)\right] & =\mathrm{E}\left[I_{l}(\omega)\right] \\
& =\sum_{m=-(l-1)}^{l-1}\left(1-\frac{|m|}{l}\right) r_{l}[m] e^{-j \omega m},
\end{aligned}
$$




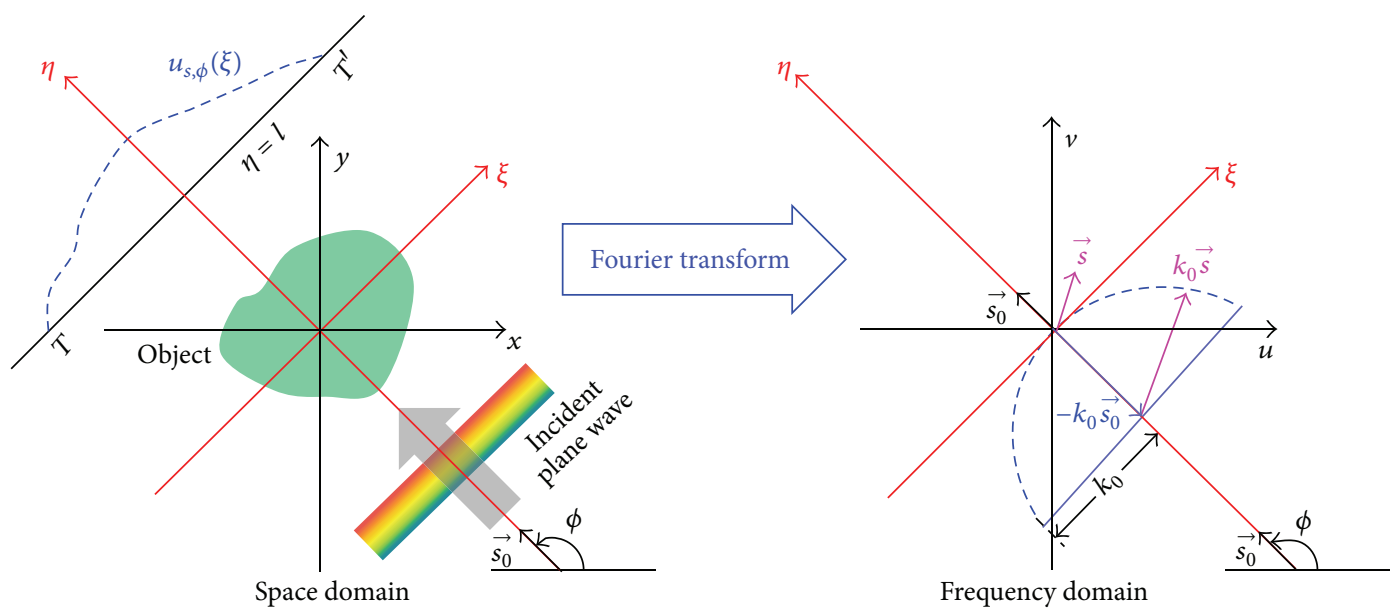

FIGURE 1: The Fourier diffraction theorem relates the Fourier transform of a diffracted projection to the Fourier transform of the object along a semicircular arc. An arbitrary object is illuminated by a plane wave propagating along the unit vector, and the coordinate system is rotated [8].

which is considered to be asymptotically unbiased as $l$ approaches infinity. Also the variance of the averaged periodogram estimate is given by [23]

$$
\begin{aligned}
\operatorname{VAR}\left[\bar{I}_{l, K}(\omega)\right] & =\frac{1}{K} \operatorname{VAR}\left[I_{l}(\omega)\right] \\
& \simeq \frac{1}{K} S_{x}(\omega)^{2} .
\end{aligned}
$$

The variance of the averaged periodogram estimate is inversely proportional to the number of iid periodograms $K$, and consequently the variance approaches zero as $K$ approaches infinity. We use (6) and (7) to conclude that the expected value remains unchanged, but only the variance of white Gaussian noise decreases for averaging $K$ iid periodogram estimates. Increasing the number of $K$ in averaging periodogram estimate truly flattens the spectral density, and the successful tomographic image can be achieved by transmitting $K$ multiple random noise waveforms with a large sequence size $l$. For the numerical simulations performed in this paper, a total of 10 iid random noise waveforms are transmitted, and each iid noise waveform is generated with 500 random amplitude samples drawn from $\mathcal{N}\left(0, \sigma^{2}\right)$. The tomographic image is formed based on the dataset from 41 discrete frequencies chosen uniformly within X-band from $8 \mathrm{GHz}$ to $10 \mathrm{GHz}$ in steps of $50 \mathrm{MHz}$.

\section{Formulation}

In this section, the scattering properties for two-dimensional cylindrical impenetrable conducting object in the bistatic scattering arrangement are discussed, and the Fourier diffraction tomography algorithm is applied to reconstruct the image of the object based on the bistatic scattering properties. The Fourier diffraction theorem has been extensively applied in the area of acoustical imaging [27-29]. The goal of diffraction tomography is to reconstruct the properties of a slice of an object from the scattered field. For planar geometry, an object is illuminated with a plane wave, and the scattered

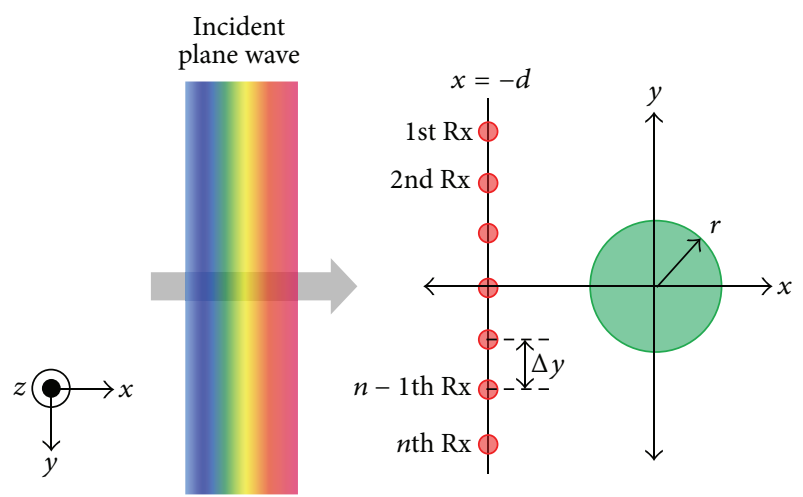

FIgURE 2: Two-dimensional backward scattering geometry for a cylindrical conducting object. Red dots and green circle represent a linear receiving array and the PEC cylinder, respectively.

fields are calculated or measured over a straight line parallel to the incident plane wave. The mathematical formulation and proof of validity of the Fourier diffraction theorem shown in Figure 1 are not presented in this section since they were already stated and published [30].

Two-dimensional backward scattering geometry for a cylindrical conducting object is shown in Figure 2. Prior research on microwave imaging has already proven that the image reconstructed in the backward scattering case is better than that obtained in the forward scattering case, based on numerical results [31].

As shown in Figure 2, a single cylindrical perfect electric conductor (PEC) object is located at the center of the simulation scene. The height of the cylindrical object is assumed to be infinitely long along the $z$-axis and the incident $z$-polarized plane wave is illuminated from $-x$ direction. The receiver spacing, $\Delta y$, is calculated to avoid aliasing effect and is given by

$$
\Delta y \leq \frac{\lambda_{\min }}{2}
$$


where $\lambda_{\min }$ is the minimum wavelength. A linear receiving array is located at $x=-d$ from the center of the cylinder object, collecting scattered field for reconstruction of the object image. The frequency sampling interval, $\Delta f$, is also considered to ensure an image that is free of false aliases and is given by

$$
\Delta f \leq \frac{c}{2 \cdot r}
$$

where $c$ is the speed of wave propagation in free space, and $r$ is the radius of the cylindrical PEC object.

As shown in the previous section, the frequency response of the transmitted white Gaussian noise waveform shows that the field amplitude is a constant nonzero amplitude value for all possible frequencies. Thus, the $z$-polarized incident plane wave of a single transmitted white Gaussian noise waveform for $N$ discrete frequencies takes the form

$$
\begin{aligned}
E_{\text {inc }}(\vec{r}) & =\widehat{z} \cdot\left(E_{1} e^{-j k_{1} \hat{x} \cdot \vec{r}}+E_{1} e^{-j k_{2} \hat{x} \cdot \vec{r}}+\cdots+E_{N} e^{-j k_{N} \hat{x} \cdot \vec{r}}\right) \\
& =\widehat{z} \cdot \sum_{n=1}^{N} E_{n} e^{-j k_{n} \hat{x} \cdot \vec{r}}
\end{aligned}
$$

where $k_{n}=\omega_{n} / c$ is the wavenumber, and $E_{n}$ is the field amplitude of the transmitted white Gaussian noise waveform at each discrete frequency of interest. The field amplitude of the noise waveform $E_{n}$ becomes the nonzero constant value as the sequence of the noise waveform approaches infinity.

We start the analysis from a single frequency and develop the process for $N$ multiple frequencies by summing up the analysis results for $N$. Also the entire analysis must be repeated for $K$ times when $K$ multiple iid noise waveforms are transmitted. The $z$-polarized incident waveform is defined as

$$
\begin{gathered}
E_{\text {inc }}(\vec{r})=\widehat{z} \cdot E_{n} e^{-j k_{n} \hat{x} \cdot \vec{r}}, \\
H_{\text {inc }}(\vec{r})=-\widehat{y} \cdot \frac{1}{\eta} \cdot E_{n} e^{-j k_{n} \widehat{x} \cdot \vec{r}},
\end{gathered}
$$

where $\eta=\sqrt{\mu_{0} / \varepsilon_{0}}$ is the intrinsic impedance in free space. If the object consists of a material having a certain dielectric constant value, the equivalent electric current distribution, $J_{\text {eq }}$, is calculated for the scattered field. The object is defined as PEC so that the scattered field observed at the linear receiving array in the $y$-direction located at $x=-d$ is calculated by applying the physical optics approximation and is given by

$$
\begin{aligned}
& E_{\text {scat,single }}\left(k_{n}, x=-d, y\right) \\
& =-j \omega \mu_{0} \int_{S} J_{\mathrm{eq}} \cdot G\left(\vec{r}-\vec{r}^{\prime}\right) d \vec{r}^{\prime} \\
& =-j k_{n} \eta \int_{S}\left(2 \widehat{n}\left(\vec{r}^{\prime}\right) \times H_{\mathrm{inc}}\left(\vec{r}^{\prime}\right)\right) \cdot G\left(\vec{r}-\vec{r}^{\prime}\right) d \vec{r}^{\prime},
\end{aligned}
$$

where $S$ is the boundary of scatterer, $\widehat{n}\left(\vec{r}^{\prime}\right)$ is the outward unit normal vector to $S$, and $G\left(\vec{r}-\vec{r}^{\prime}\right)$ is Green's function for twodimensional geometry defined as

$$
G\left(\vec{r}-\vec{r}^{\prime}\right)=-\frac{j}{4} H_{0}^{(2)}\left(k_{n}\left(\vec{r}-\vec{r}^{\prime}\right)\right)
$$

where $H_{0}^{(2)}$ is the zeroth-order Hankel function of the second kind.

Similarly, for the case of discrete scattering objects, the scattered field of $p$ objects over a linear array in the $y$ direction located at $x=-d$ is expressed as

$$
E_{\text {scat,multiple }}\left(k_{n}, x=-d, y\right)=\sum_{p} E_{\text {scat, single }}(p) .
$$

On assuming the polarization in the $z$-direction, the general form of scattered field, $u_{\text {scat }}$, obtained by the receivers at $x=-d$ becomes

$$
\begin{aligned}
u_{\text {scat }} & \left(k_{n}, x=-d, y\right) \\
& =\widehat{z} \cdot E_{\text {scat }}\left(k_{n}, x=-d, y\right) \\
& =-j k_{n} E_{n} \iint o_{\text {scat }}\left(\vec{r}^{\prime}\right) e^{-j k_{n} \cdot \vec{x}^{\prime}} G\left(\vec{r}-\vec{r}^{\prime}\right) d^{2} \vec{r}^{\prime},
\end{aligned}
$$

where

$$
o_{\text {scat,single }}\left(\vec{r}^{\prime}\right)=-2 \widehat{n}\left(\vec{r}^{\prime}\right) \cdot \widehat{x} \delta\left(S\left(\vec{r}^{\prime}\right)\right)
$$

is defined as the scattering object function of a single PEC object which is related to the object shape, and $\delta\left(S\left(\vec{r}^{\prime}\right)\right)$ is a Dirac delta function defined as

$$
\delta\left(S\left(\vec{r}^{\prime}\right)\right) \begin{cases}=0 & \text { as } \vec{r}^{\prime} \in S \\ \neq 0 & \text { elsewhere. }\end{cases}
$$

Similarly, the scattering function shown in (16) for discrete scattering objects can be written as

$$
o_{\text {scat,multiple }}\left(\vec{r}^{\prime}\right)=\sum_{p} o_{\text {scat,single }}(p) .
$$

By using the plane wave expansion of Green's function [32], the one-dimensional Fourier transform of $u_{\text {scat }}$, defined in (15), in $y$-direction can be written as

$$
\widetilde{U}_{\text {scat }}\left(k_{n}, x=-d, k_{y}\right)=\frac{k_{n}^{2} E_{n}}{j 2 \gamma} e^{-j \gamma d} \widetilde{O}_{\text {scat }}\left(-\gamma-k_{n}, k_{y}\right) \text {, }
$$

where

$$
\gamma= \begin{cases}\sqrt{k_{n}^{2}-k_{y}^{2}} & \text { as }\left|k_{y}\right| \leq k_{n} \\ -j \sqrt{k_{y}^{2}-k_{n}^{2}} & \text { as }\left|k_{y}\right|>k_{n} .\end{cases}
$$

If $k_{x}$ is defined as

$$
k_{x}=-\gamma-k_{n}
$$

the Fourier transform of the two-dimensional scattering object function, $\widetilde{O}_{\text {scat }}\left(k_{x}, k_{y}\right)$, defined in (19) is given by

$$
\widetilde{O}_{\text {scat }}\left(k_{x}, k_{y}\right)=\iint o_{\text {scat }}(x, y) e^{-j\left(k_{x} x+k_{y} y\right)} d x d y .
$$

In this case, the arguments of $\widetilde{O}_{\text {scat }}\left(k_{x}, k_{y}\right)$ are related by

$$
\left(k_{x}+k_{n}\right)^{2}+k_{y}^{2}=k_{n}^{2} .
$$



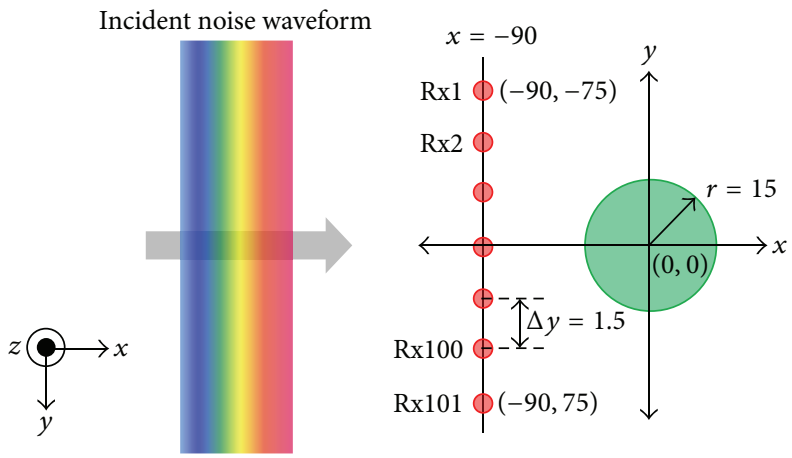

FIgURE 3: Two-dimensional backward scattering simulation geometry for a cylindrical conducting object. A single PEC cylinder with a radius of $15 \mathrm{~cm}$ is located at $(0,0)$, and a linear receiving array is located $90 \mathrm{~cm}$ away from the origin in $-x$ direction.

Equations (19) and (23) show that as a two-dimensional scattering object is illuminated by a plane wave at the frequency $\omega_{n}$, the one-dimensional Fourier transform of the scattered field yields a semicircle centered at $\left(0,-k_{n}\right)$ with radius $k_{n}$ in the two-dimensional Fourier space, which relates to the Fourier diffraction theorem depicted in Figure 1. By using $N$ number of frequencies, the radius in the two-dimensional Fourier space changes, which enhances the resolution and accuracy of the image. Also the variance of the frequency response is reduced by taking average of the frequency responses of $K$ multiple transmissions of iid UWB noise waveforms $[8,33]$.

\section{Numerical Simulation Results}

Based on the formulation described in previous section, this section discusses the numerical simulation results of diffraction tomography using band-limited iid UWB WGN waveforms for various scattering target geometries.

4.1. Diffraction Tomography with a Single Transmitted WGN Waveform for a Single Scattering Object. As shown in Figure 3, two-dimensional backward scattering geometry with a single cylindrical conducting object is simulated with two band-limited iid UWB WGN waveforms. The cylindrical PEC object with a radius of $15 \mathrm{~cm}$ is located at the origin, and the cylinder is assumed to be infinitely long along the $z$-axis.

The scattered field is uniformly sampled at receiving array Rx1 through Rx101 with frequency swept within X-band from $8 \mathrm{GHz}$ to $10 \mathrm{GHz}$ in 41 steps of $50 \mathrm{MHz}$. The locations of Rxl and Rx101 are at $(-90 \mathrm{~cm},-75 \mathrm{~cm})$ and $(-90 \mathrm{~cm}$, $75 \mathrm{~cm}$ ), respectively, and the receiver spacing, $\Delta y$, is set to $1.5 \mathrm{~cm}$ based on (8) when $f_{\max }$ is $10 \mathrm{GHz}$. The maximum frequency stepping interval, $\Delta f$, is also calculated using (9), which yields a value of $1 \mathrm{GHz}$; however, $50 \mathrm{MHz}$ frequency stepping interval enhances the quality of tomographic image compared to the maximum frequency stepping interval of $1 \mathrm{GHz}$

For two band-limited iid UWB WGN transmitted waveforms, each iid WGN waveform is generated with 500 random amplitude samples drawn from $\mathscr{N}\left(0, \sigma^{2}\right)$, which are shown in Figures 4 and 5, to reconstruct tomographic images of the cylindrical PEC object based on the scattered field observed at Rx1 through Rx101.

A block diagram shown in Figure 6 displays the tomographic image reconstruction method using diffraction tomography. The one-dimensional Fourier transformed scattered field data collected at the receiving array Rxl through Rx101 is Fourier transformed into two-dimensional object Fourier space data, $\widetilde{O}\left(k_{x}, k_{y}\right)$, by using (19). Such Fourier space data is two-dimensional inverse Fourier transformed to obtain the scattering object function, $o(x, y)$.

Fourier space data and the tomographic images of the single cylindrical conducting object, using the first UWB WGN waveform shown in Figure 4(a), are calculated and displayed in Figures 7(a) and 7(b), respectively. The tomographic image of the single PEC cylinder appears to be successfully reconstructed using the first UWB WGN transmitted waveform.

Similarly, both Fourier space data and the tomographic images of the single PEC cylinder using the second UWB WGN waveform shown in Figure 5(a) are also shown in Figures 8(a) and 8(b), respectively. In this case, the tomographic image is significantly affected by the unexpected notch observed in the frequency spectrum at $9.1 \mathrm{GHz}$ in Figure 5(b), so that the tomographic image of the object cannot be achieved correctly compared to the previous case where the first WGN waveform is transmitted. Based on the simulation results with two band-limited iid UWB WGN waveforms, a single transmission of WGN waveform may or may not be sufficient to reconstruct a successful tomographic image of the object with diffraction tomography algorithm due to the undesired and unexpected notches in the spectral density of the practical UWB WGN transmitted waveforms.

\subsection{Diffraction Tomography with Multiple Transmitted iid} WGN Waveforms for a Single Scattering Object. Figure 9 shows $K$ iid UWB WGN waveforms being transmitted to reconstruct a final image of the object in order to bypass a shortcoming of the single transmission of WGN waveform displayed in Figure 8(b). The proposed imaging method with multiple iid WGN transmitted waveforms is established based on the method of the WGN periodogram averaging described in Section 2. 


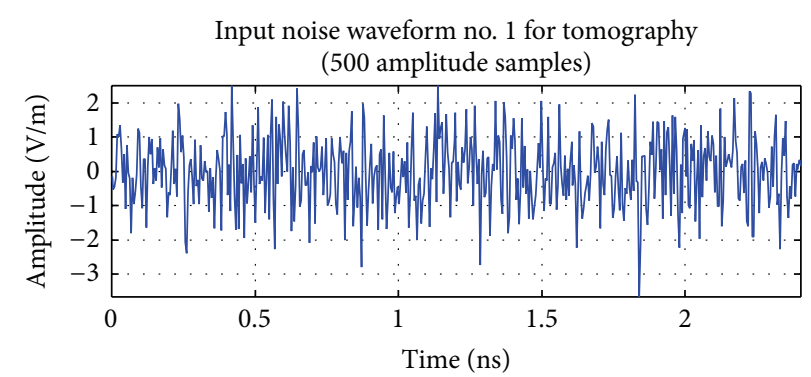

(a)

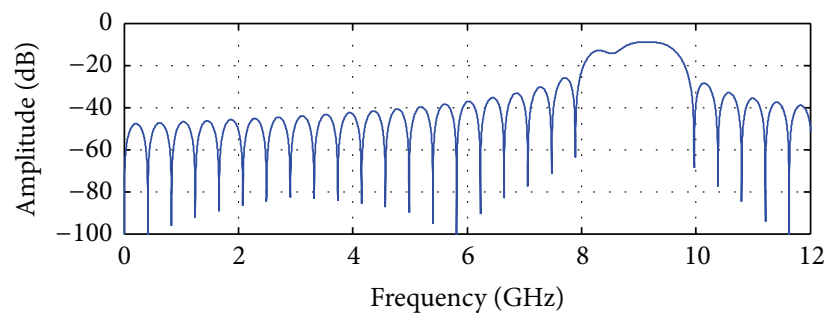

(b)

FIGURE 4: (a) The first band-limited UWB WGN transmitted waveform generated with 500 amplitude samples $(l=500)$ drawn from $\mathcal{N}\left(0, \sigma^{2}\right)$. Pulse duration is $2.4 \mathrm{~ns}$. (b) The frequency spectrum of the time domain WGN waveform shown in Figure 4(a). The frequency ranges are shown from DC to $12 \mathrm{GHz}$ only [8].

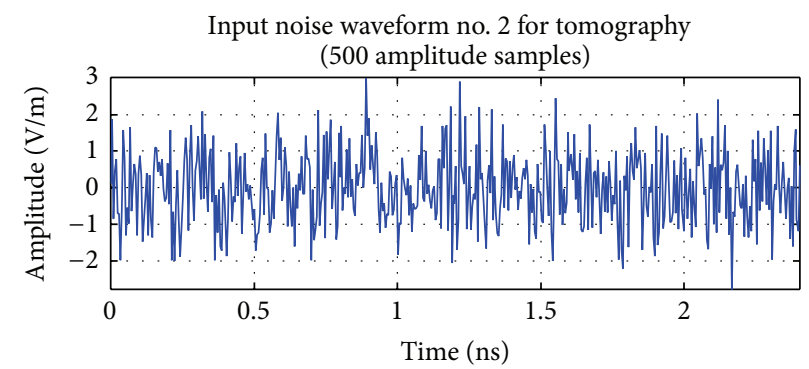

(a)

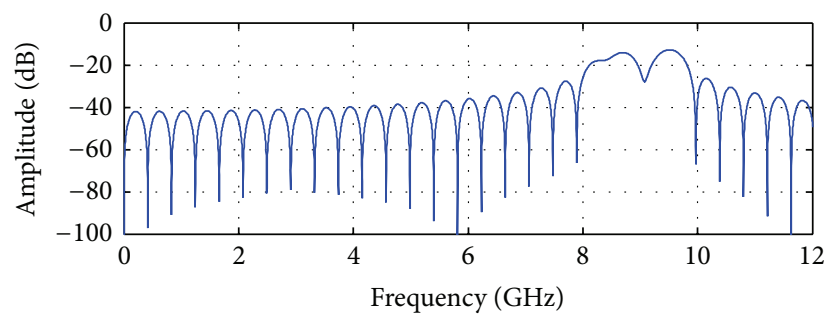

(b)

FIGURE 5: (a) The second band-limited UWB WGN transmitted waveform generated with 500 amplitude samples $(l=500)$ drawn from $\mathscr{N}\left(0, \sigma^{2}\right)$. Pulse duration is $2.4 \mathrm{~ns}$. (b) The frequency spectrum of the time domain WGN waveform is shown in Figure $5(\mathrm{a})$. The frequency ranges are shown from $\mathrm{DC}$ to $12 \mathrm{GHz}$ only [8].

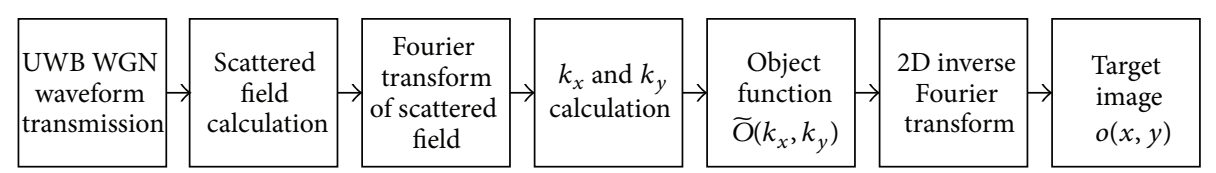

FIGURE 6: The image reconstruction method using diffraction tomography [8]. 


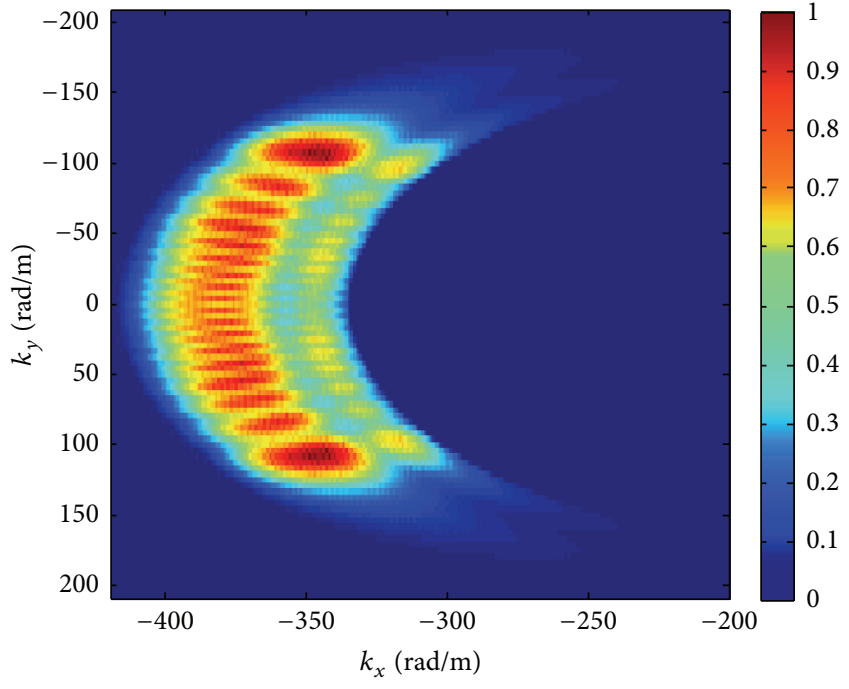

(a)

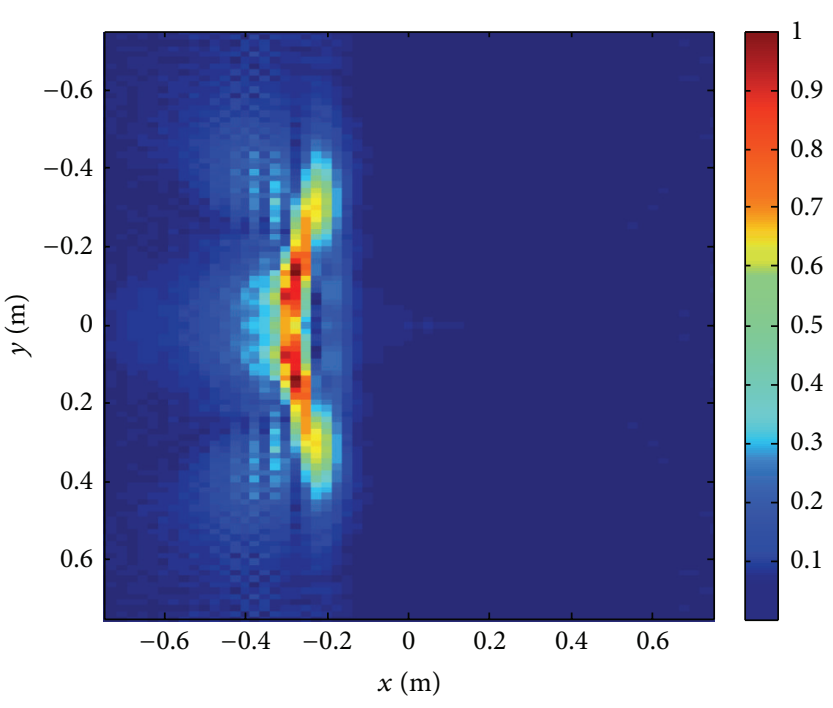

(b)

FIgURE 7: (a) The normalized magnitude of Fourier space data of the single cylindrical conducting object. The colorbar indicates the normalized magnitude of $\widetilde{O}\left(k_{x}, k_{y}\right)$. (b) The tomographic image using the first UWB WGN waveform shown in Figure 4(a). The colorbar indicates the normalized magnitude of scattering object function, $o(x, y)$.

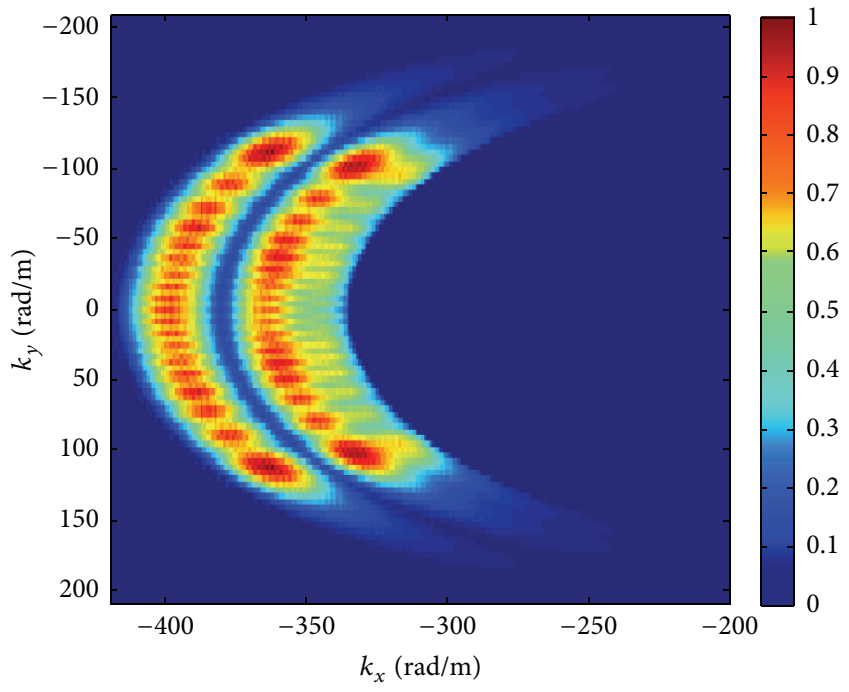

(a)

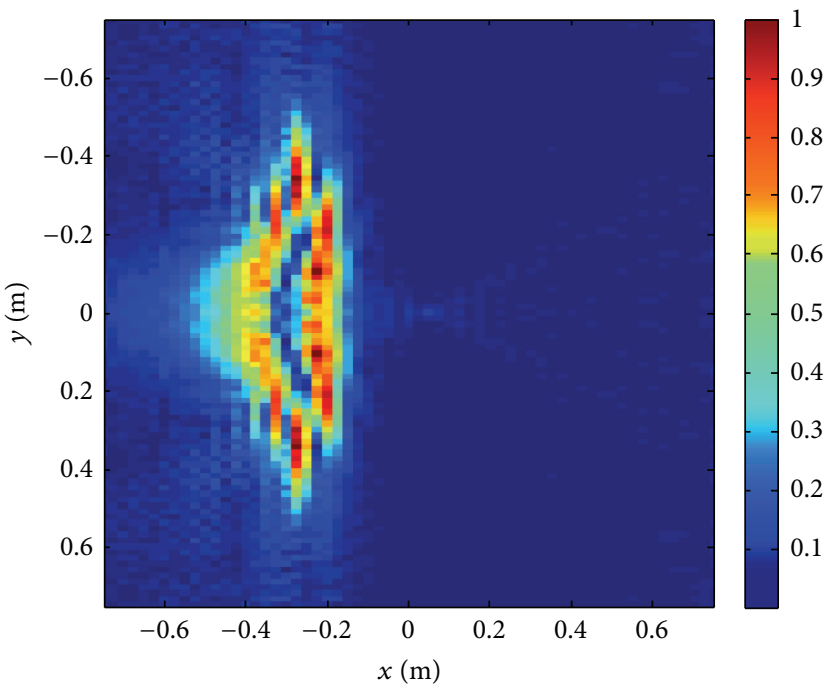

(b)

Figure 8: (a) The normalized magnitude of Fourier space data of the single cylindrical conducting object. The colorbar indicates the normalized magnitude of $\widetilde{O}\left(k_{x}, k_{y}\right)$. (b) The tomographic image using the second UWB WGN waveform shown in Figure 5(a). The colorbar indicates the normalized magnitude of scattering object function, $o(x, y)$.

As shown in Figure 9, the final tomographic image of the scattered object is reconstructed via sum and average of all $K$ discrete images for $K$ th band-limited iid UWB WGN transmitted waveforms. For scattered field and diffraction tomography simulations with $K$ multiple iid WGN waveforms, 10 iid UWB WGN waveforms over a frequency range from 8 to $10 \mathrm{GHz}$ are generated with 500 random amplitude samples drawn from $\mathscr{N}\left(0, \sigma^{2}\right)$ and transmitted for backward scattering field data.
Figures 10(a), 10(b), 10(c), and 10(d) display the four final tomographic images when one, three, seven and all ten discrete images are summed and averaged, respectively. Successful tomographic imaging of the target is achieved after averaging all ten images by visual inspection of the formed images as shown in Figure 10(d), and increasing the number of transmissions of the iid UWB WGN waveform tends to enhance the quality of final tomographic image of the object by reducing the variance of the spectral response of WGN. 


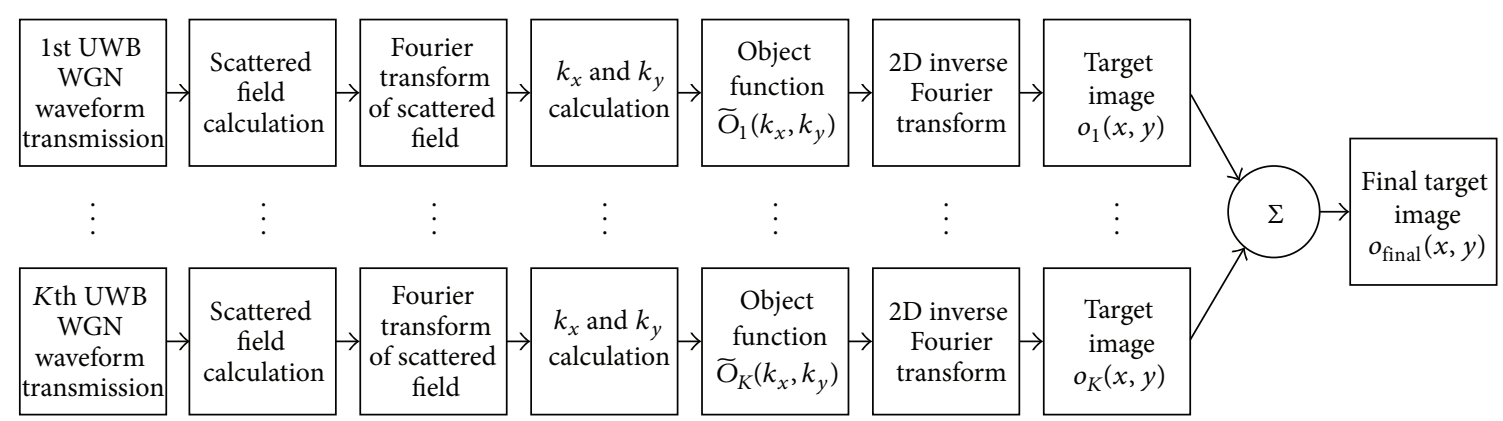

FIGURE 9: The image reconstruction method with $K$ multiple iid WGN transmitted waveforms using diffraction tomography.

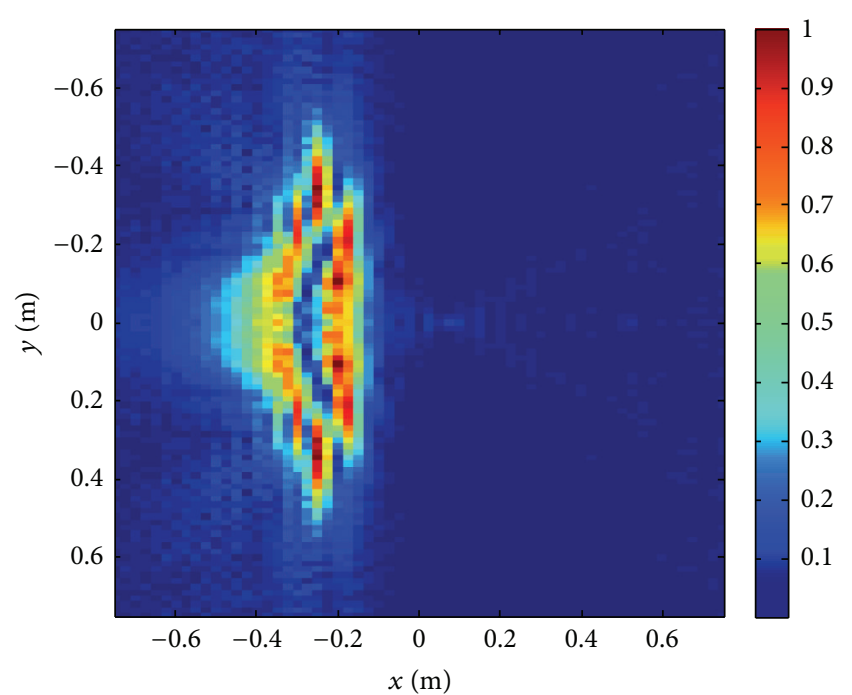

(a)

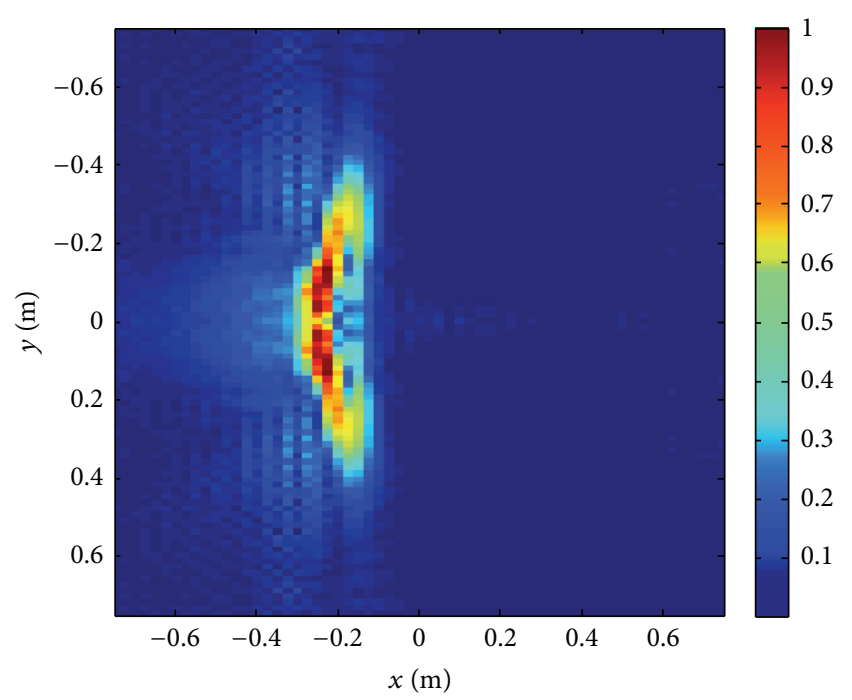

(c)

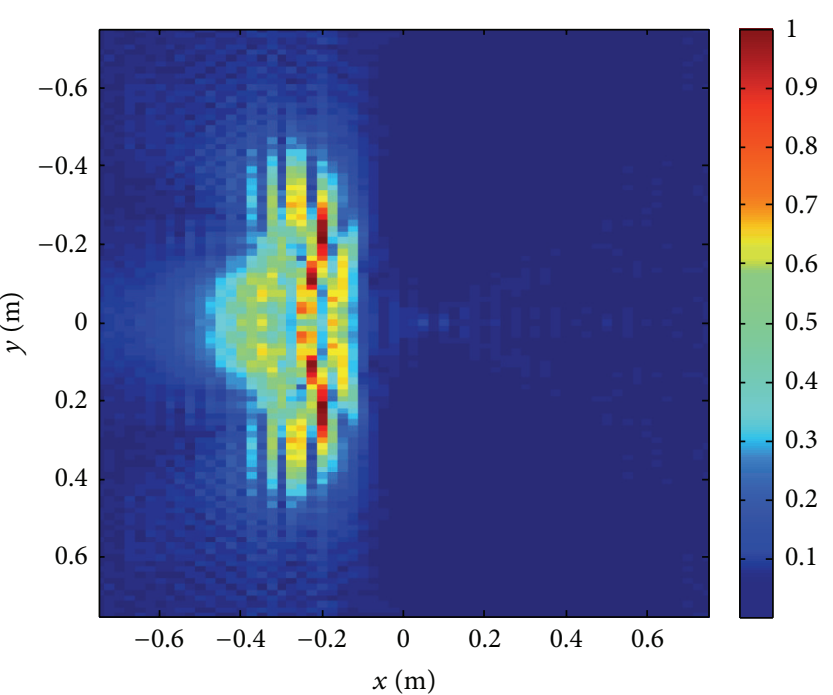

(b)

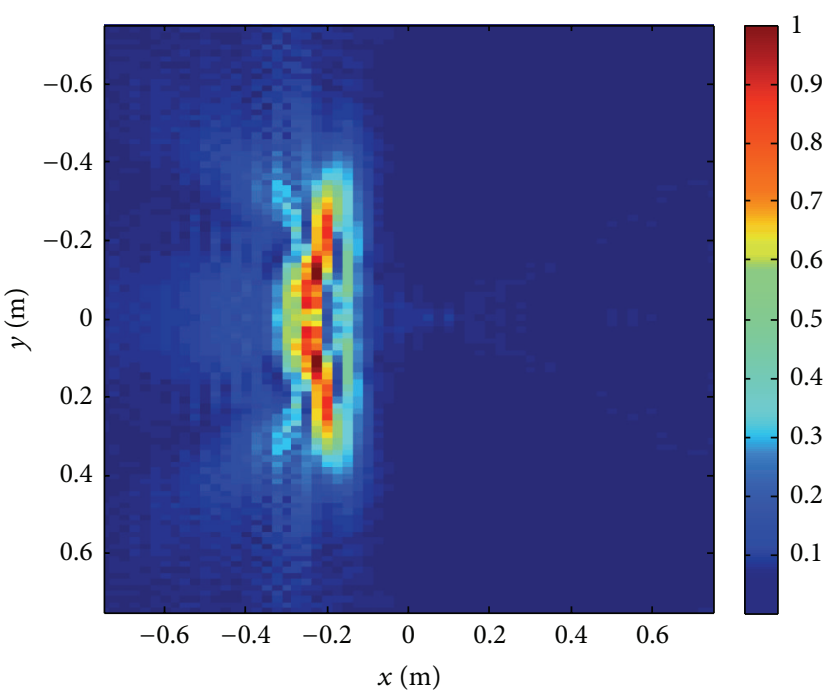

(d)

FIGURE 10: The final tomographic image of a single PEC cylinder located at $(0,0)$ after summing and averaging process with the (a) one transmitted WGN waveform image, (b) three transmitted WGN waveform images, (c) seven transmitted WGN waveform images, and (d) all ten transmitted WGN waveform images. The colorbar indicates the normalized magnitude of scattering object function, $o(x, y)$. 


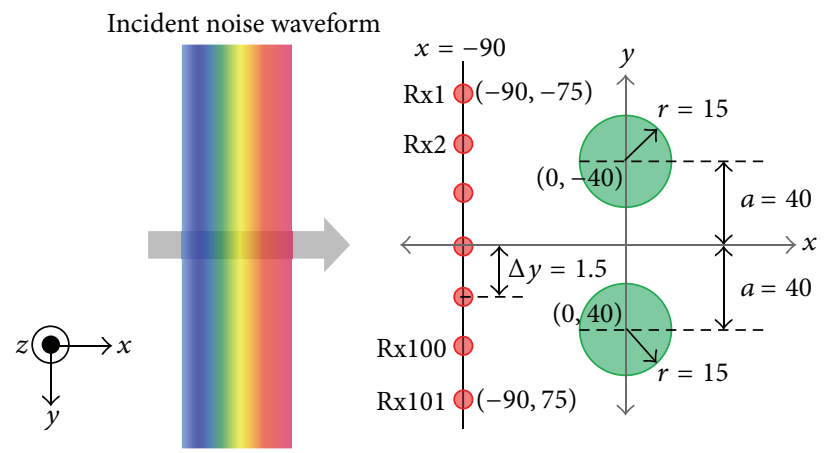

FIGURE 11: Two-dimensional backward scattering simulation geometry for two symmetrically distributed cylindrical conducting objects. PEC cylinders with radii of $15 \mathrm{~cm}$ are located at $(0 \mathrm{~cm},-40 \mathrm{~cm})$ and $(0 \mathrm{~cm}, 40 \mathrm{~cm})$, and a linear receiving array is located $90 \mathrm{~cm}$ away from the origin in $-x$ direction.

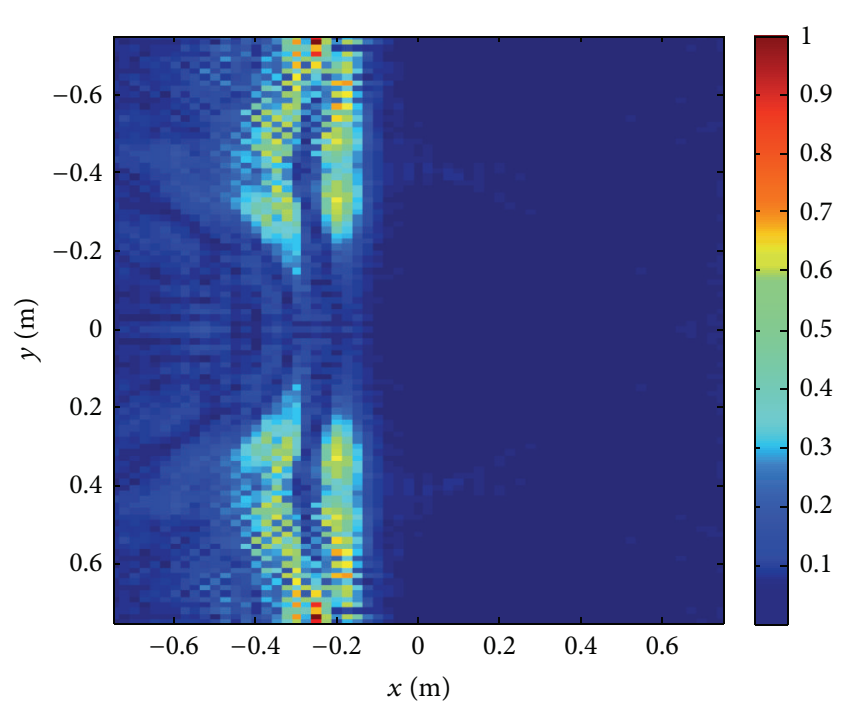

(a)

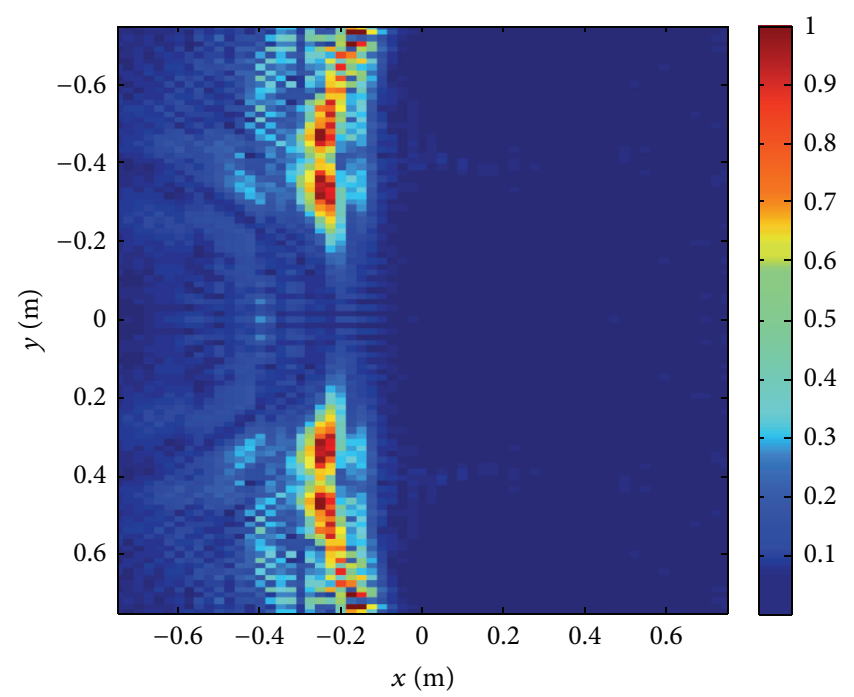

(c)

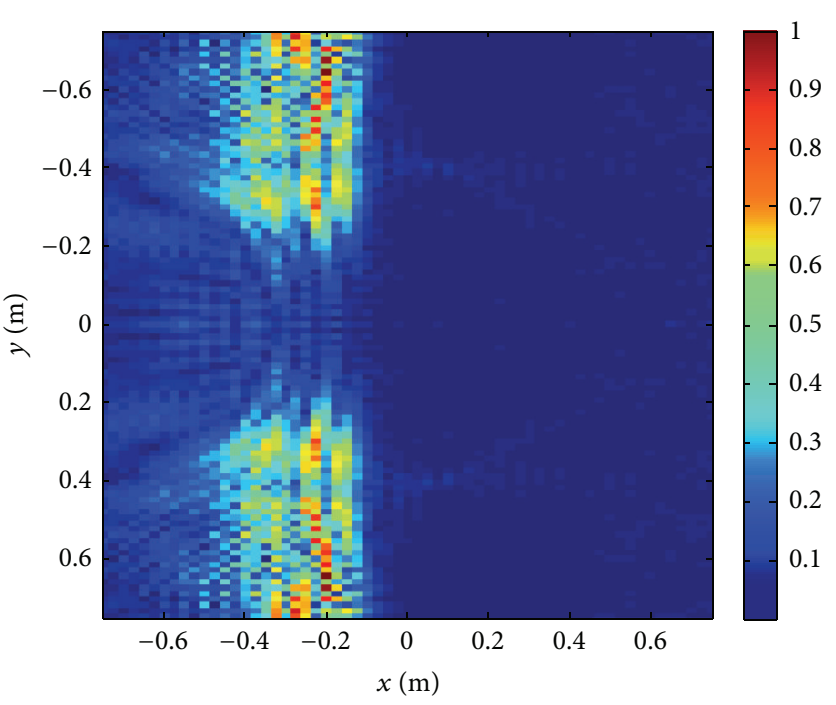

(b)

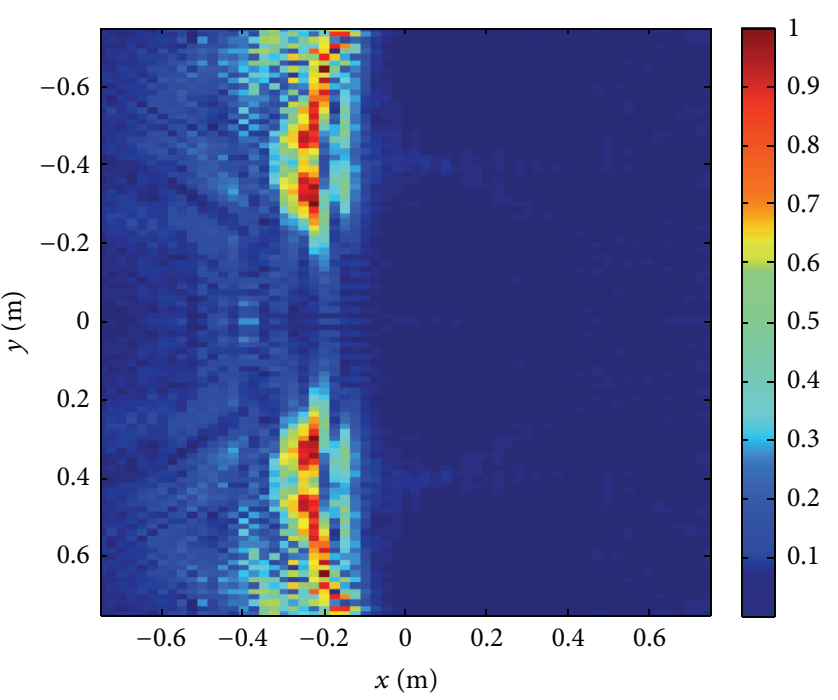

(d)

FIGURE 12: The final tomographic image of two symmetrically distributed PEC cylinders located at $(0 \mathrm{~cm},-40 \mathrm{~cm})$ and $(0 \mathrm{~cm}, 40 \mathrm{~cm})$ after summing and averaging process with the (a) one transmitted WGN waveform image, (b) three transmitted WGN waveform images, (c) seven transmitted WGN waveform images, and (d) all ten transmitted WGN waveform images. 


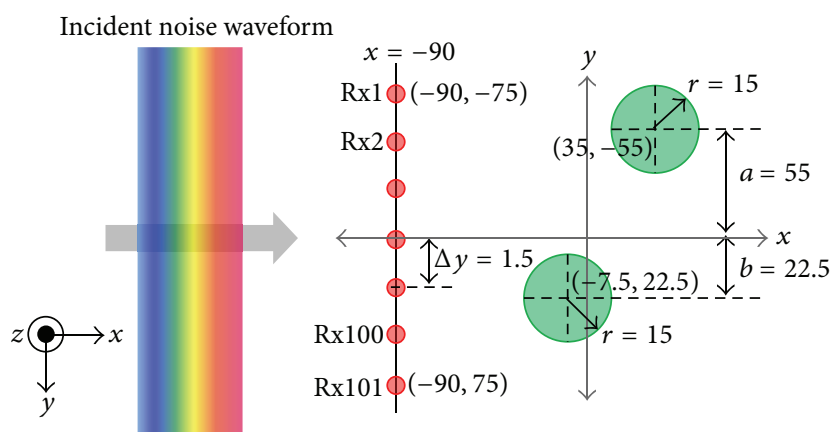

FIGURE 13: Two-dimensional backward scattering simulation geometry for two randomly distributed cylindrical conducting objects. PEC cylinders with radii of $15 \mathrm{~cm}$ are located at $(-7.5 \mathrm{~cm}, 22.5 \mathrm{~cm})$ and $(35 \mathrm{~cm},-55 \mathrm{~cm})$.

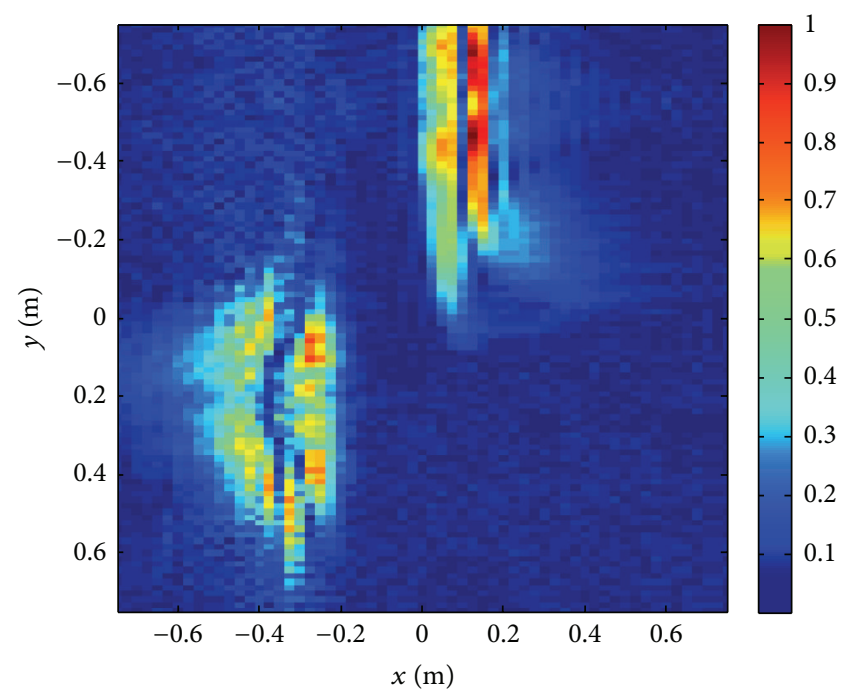

(a)

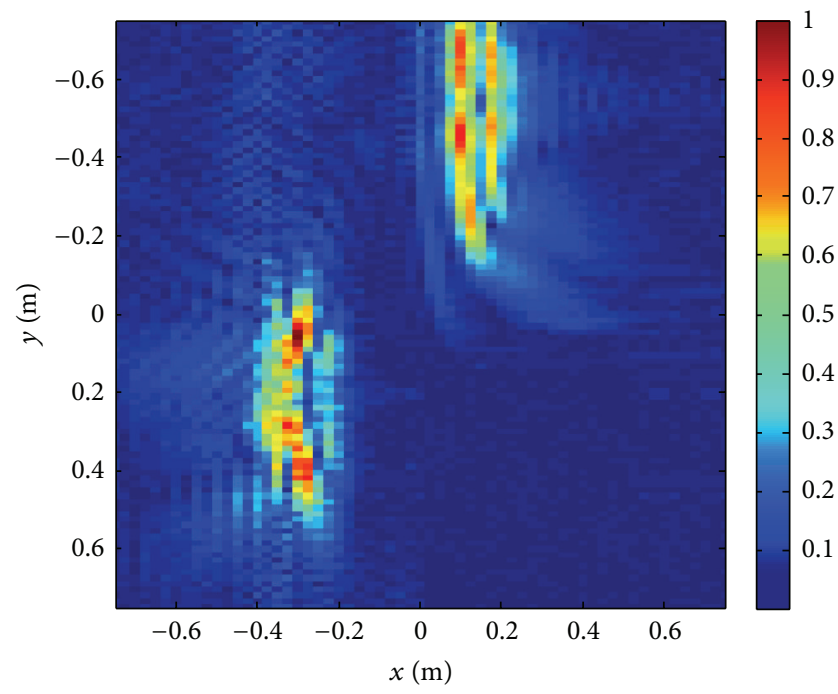

(c)

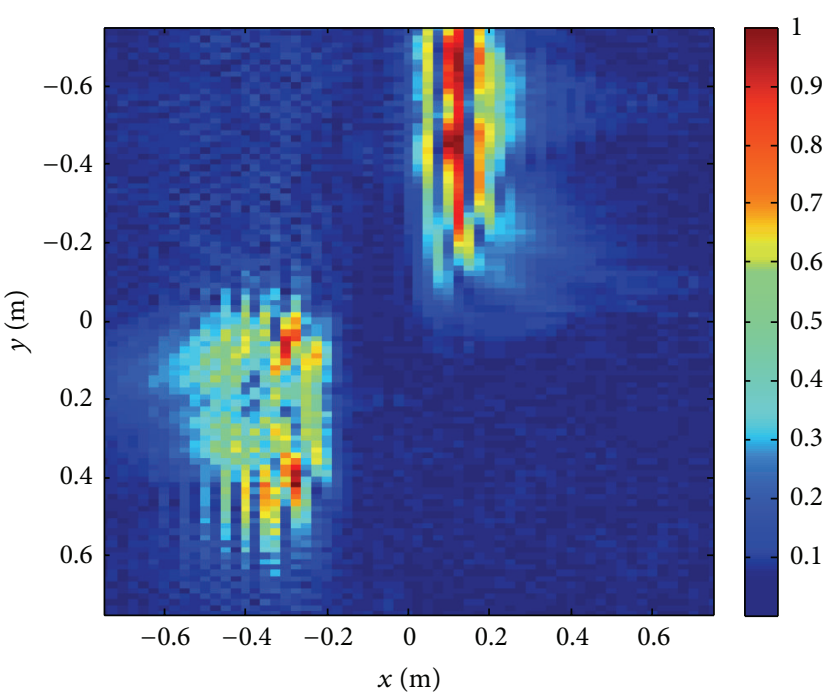

(b)

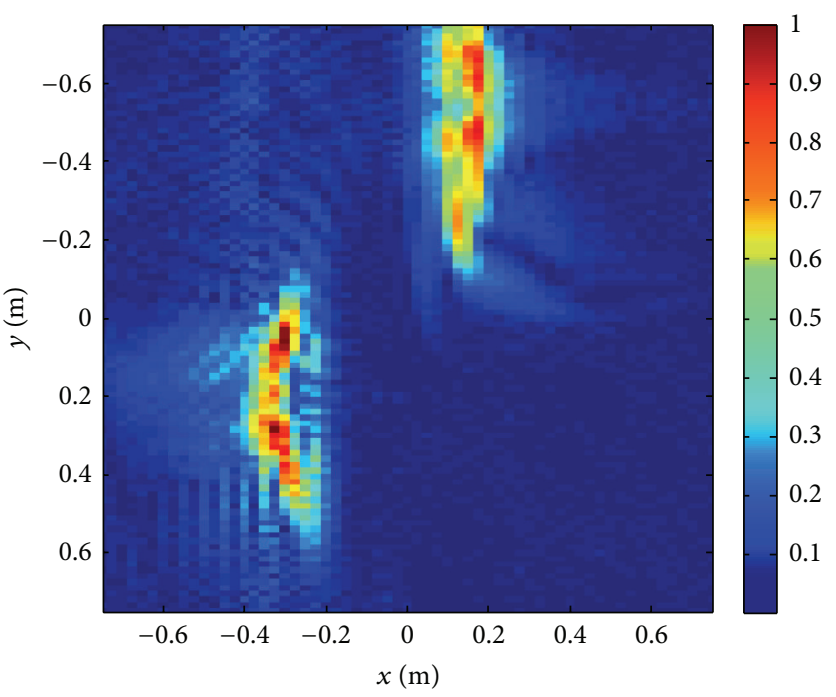

(d)

FIGURE 14: The final tomographic image of two randomly distributed PEC cylinders located at $(-7.5 \mathrm{~cm}, 22.5 \mathrm{~cm})$ and $(35 \mathrm{~cm},-55 \mathrm{~cm})$ after summing and averaging process with the (a) one transmitted WGN waveform image, (b) three transmitted WGN waveform images, (c) seven transmitted WGN waveform images, and (d) all ten transmitted WGN waveform images. 


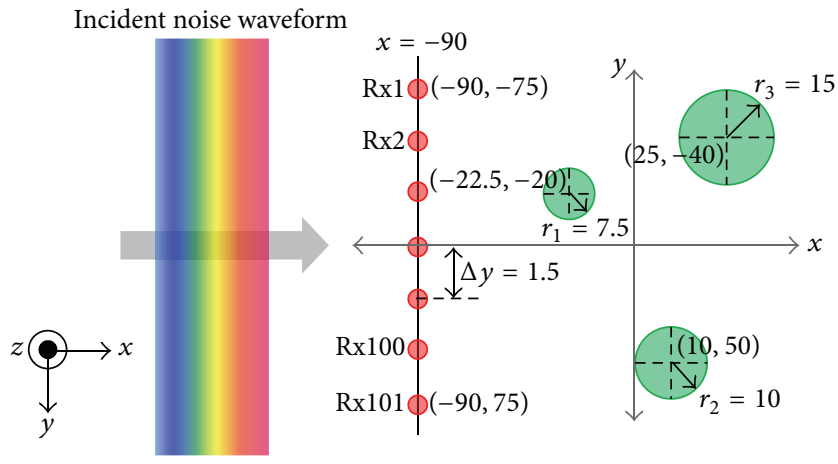

FIGURE 15: Two-dimensional backward scattering simulation geometry for three randomly distributed cylindrical conducting objects in different sizes. Three PEC cylinders with radii of $7.5 \mathrm{~cm}, 10 \mathrm{~cm}$, and $15 \mathrm{~cm}$ are located at $(-22.5 \mathrm{~cm},-20 \mathrm{~cm}),(10 \mathrm{~cm}, 50 \mathrm{~cm})$, and $(25 \mathrm{~cm}$, $-40 \mathrm{~cm})$, respectively.

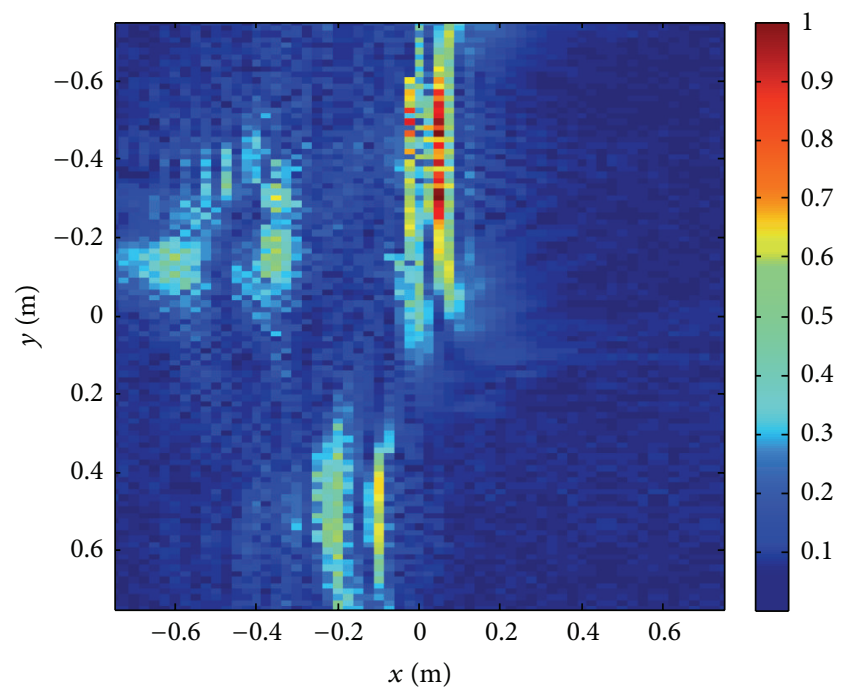

(a)

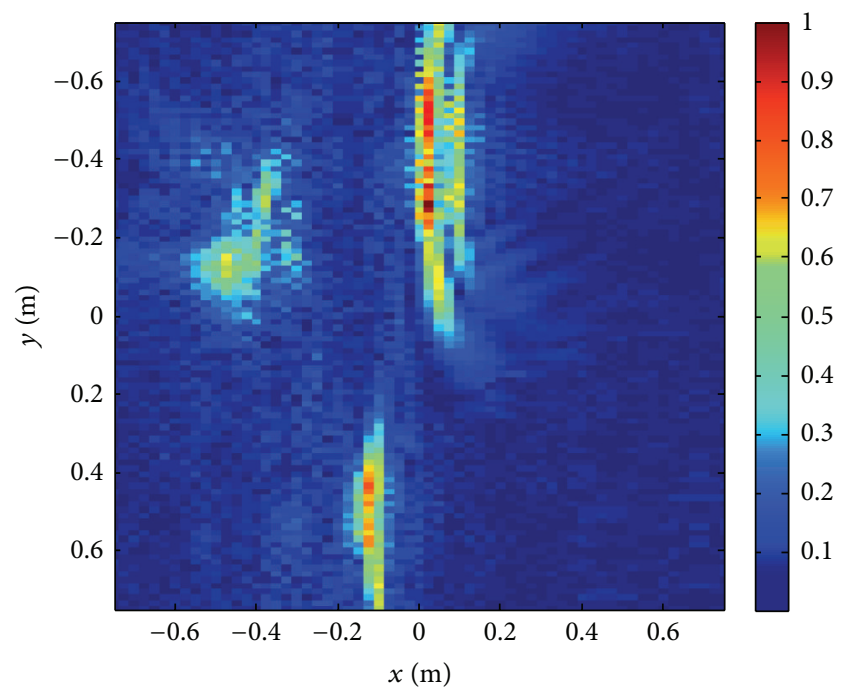

(c)

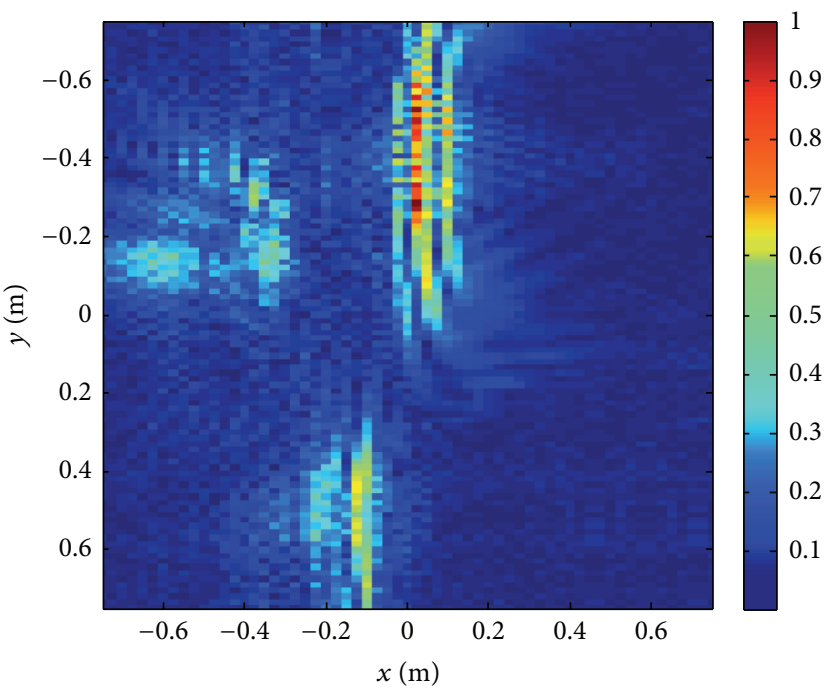

(b)

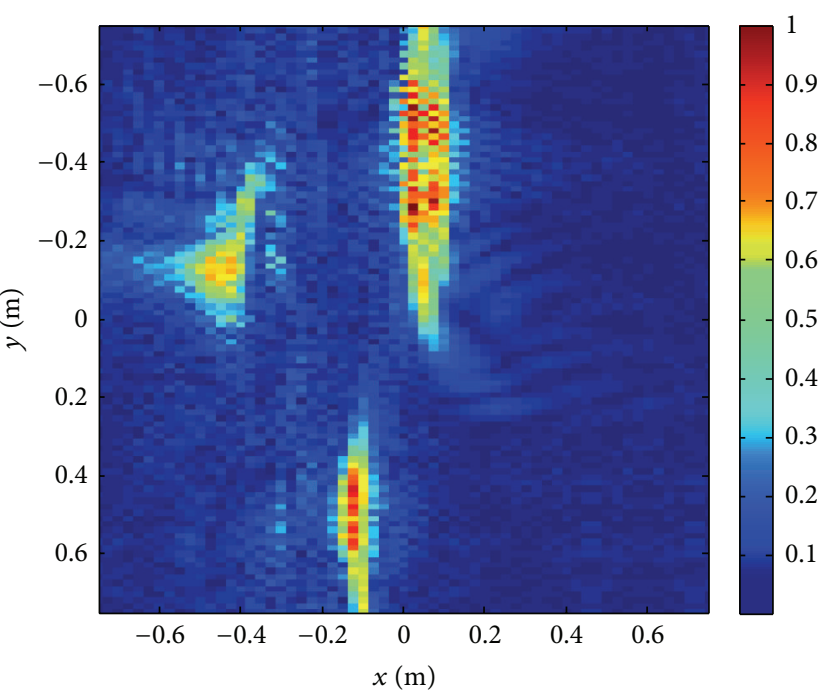

(d)

FIGURE 16: The final tomographic image of three randomly distributed PEC cylinders in different sizes after summing and averaging process with the (a) one transmitted WGN waveform image, (b) three transmitted WGN waveform images, (c) seven transmitted WGN waveform images, and (d) all ten transmitted WGN waveform images. 


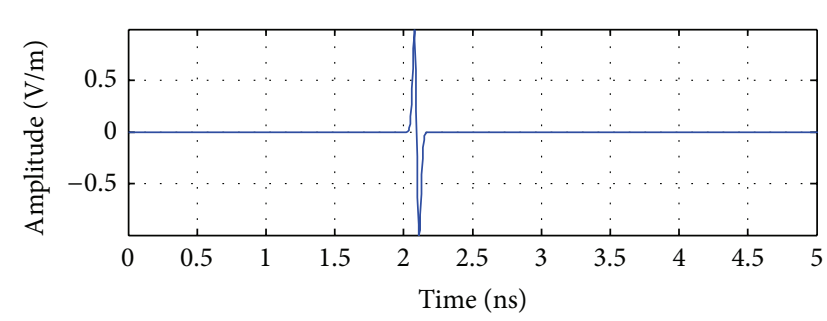

(a)

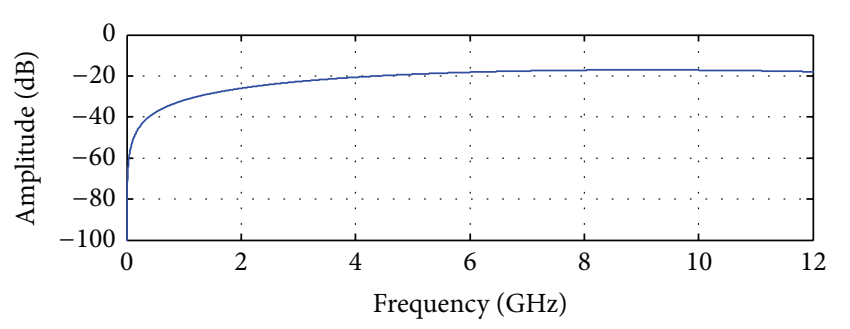

(b)

FIGURE 17: (a) The first derivative Gaussian input waveform with a pulse width of $0.1 \mathrm{~ns}$. (b) The frequency spectrum of the first derivative Gaussian input waveform shown in Figure 17(a). The frequency spectrum ranges are displayed from DC to $12 \mathrm{GHz}$ only.

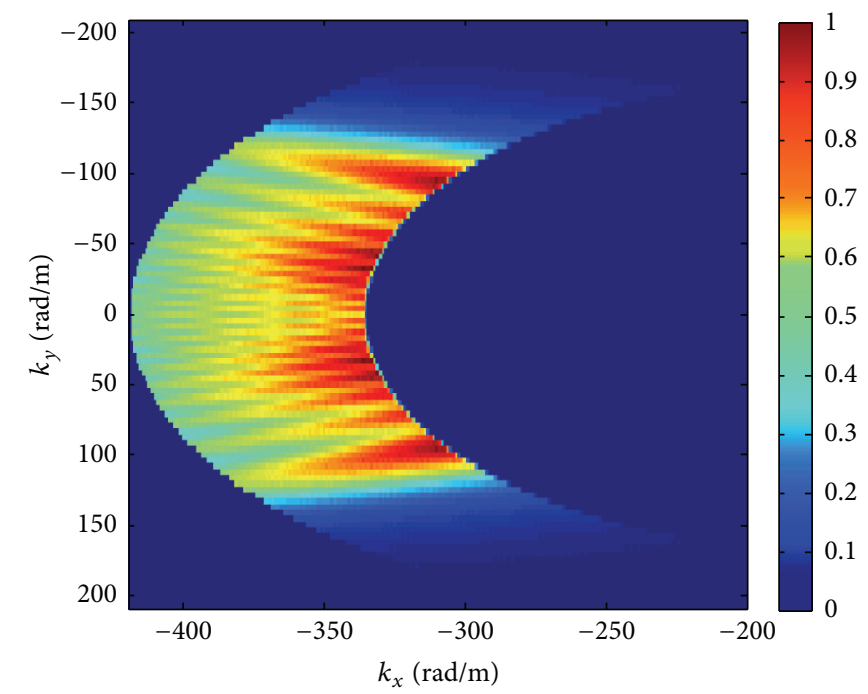

(a)

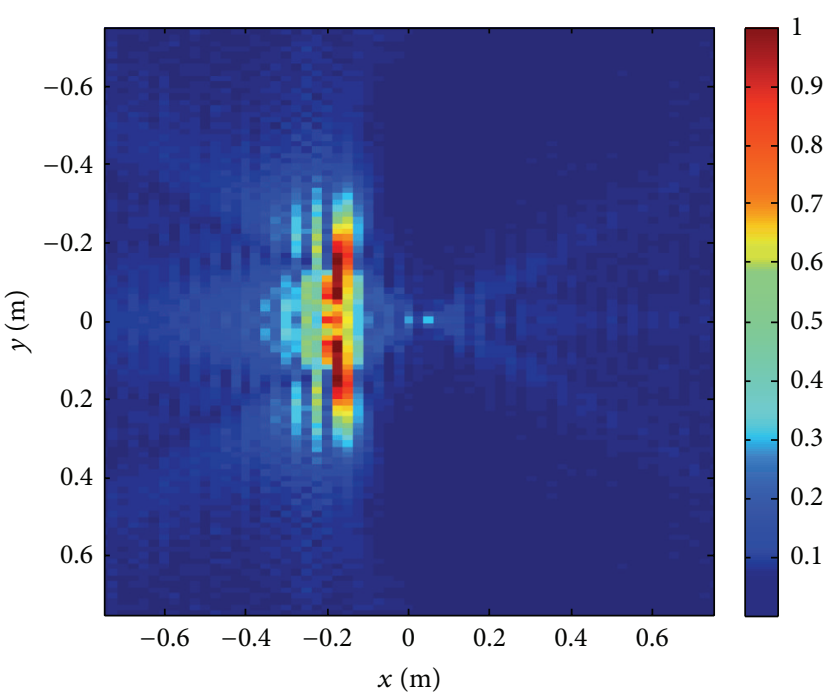

(b)

FIGURE 18: (a) The magnitude of Fourier space data based on the transmission of the first derivative Gaussian waveform shown in Figure 17(a) for the single cylindrical PEC object shown in Figure 3. The colorbar indicates the normalized magnitude of $\widetilde{O}\left(k_{x}, k_{y}\right)$. (b) The tomographic image of the single cylindrical conducting object located at $(0,0)$. The colorbar indicates the normalized magnitude of scattering object function, $o(x, y)$.

4.3. Diffraction Tomography with Multiple Transmitted iid WGN Waveforms for Two Symmetrically Distributed Scattering Objects. Based on the simulation result of the single PEC cylinder case with multiple transmitted noise waveforms, we can point out that transmitting multiple iid UWB WGN waveforms delivers the acceptable quality of image of the object for diffraction tomography.

Two-dimensional backward scattering geometry for two cylindrical conducting objects, which is shown in Figure 11, is also simulated with 10 iid UWB WGN waveforms. Two cylindrical PEC objects with radii of $15 \mathrm{~cm}$ are located at $(0 \mathrm{~cm},-40 \mathrm{~cm})$ and $(0 \mathrm{~cm}, 40 \mathrm{~cm})$ such that they are symmetrically positioned with respect to the $x$-axis. The distance from the center of each PEC cylinder to the $x$-axis, $a$, is $40 \mathrm{~cm}$, and they are also assumed to be infinitely long along the $z$ axis. The coordinates of all 101 receivers, and the receiver spacing, $\Delta y$, remained the same as shown in Figure 3. The scattered field is uniformly sampled at Rxl through Rx101 with same frequency swept within $\mathrm{X}$-band over $8-10 \mathrm{GHz}$ in 41 steps as well.

For scattered field and diffraction tomography simulations for multiple scattering objects with multiple iid WGN waveforms, the same 10 iid UWB WGN waveforms, which are used for the simulation with a single PEC object in the previous section, are transmitted in the same sequence. The scattered field due to two symmetrically positioned PEC objects is calculated based on the equations derived in Section 3, and the final tomographic image of objects is formed via the proposed image reconstruction method shown in Figure 9.

Figures 12(a), 12(b), 12(c), and 12(d) display four final tomographic images for two symmetrically distributed PEC cylinders cases when one, three, seven and all ten discrete images are summed and averaged, respectively. As shown in Figure 12(d), tomographic image of the two PEC cylinders is successfully reconstructed with multiple transmitted noise waveforms as expected. However, the mutual coupling effects 


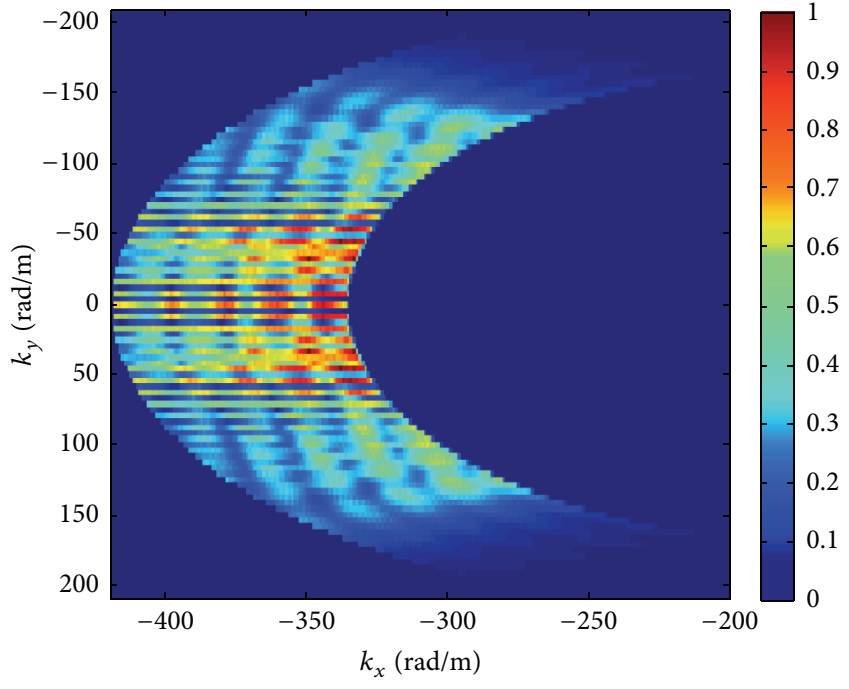

(a)

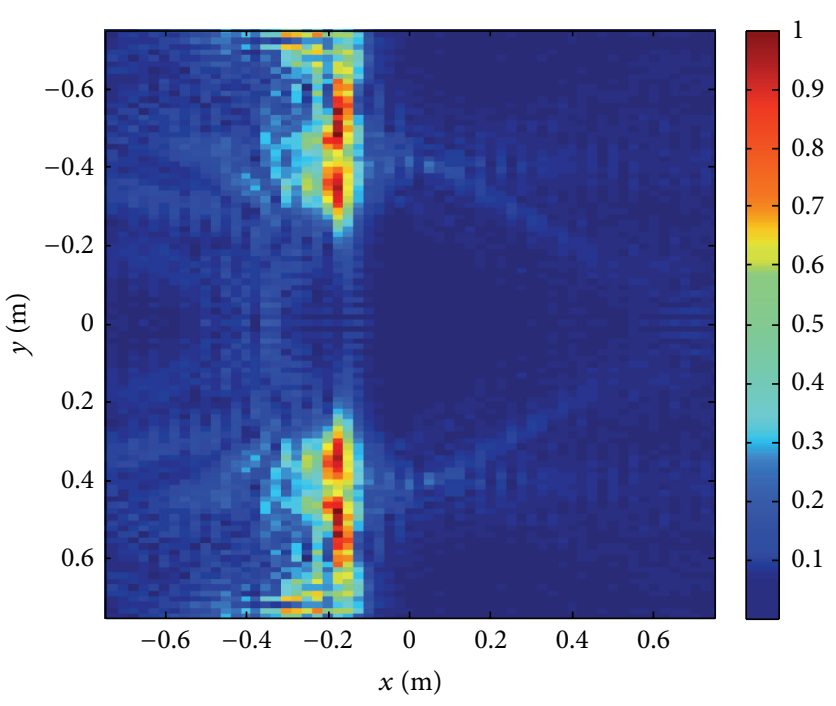

(b)

FIGURE 19: (a) The magnitude of Fourier space data based on the transmission of the first derivative Gaussian waveform shown in Figure 17(a) for two symmetrically distributed cylindrical PEC objects shown in Figure 11. (b) The tomographic image of two symmetrically distributed cylindrical conducting objects located at $(0 \mathrm{~cm},-40 \mathrm{~cm})$ and $(0 \mathrm{~cm}, 40 \mathrm{~cm})$.

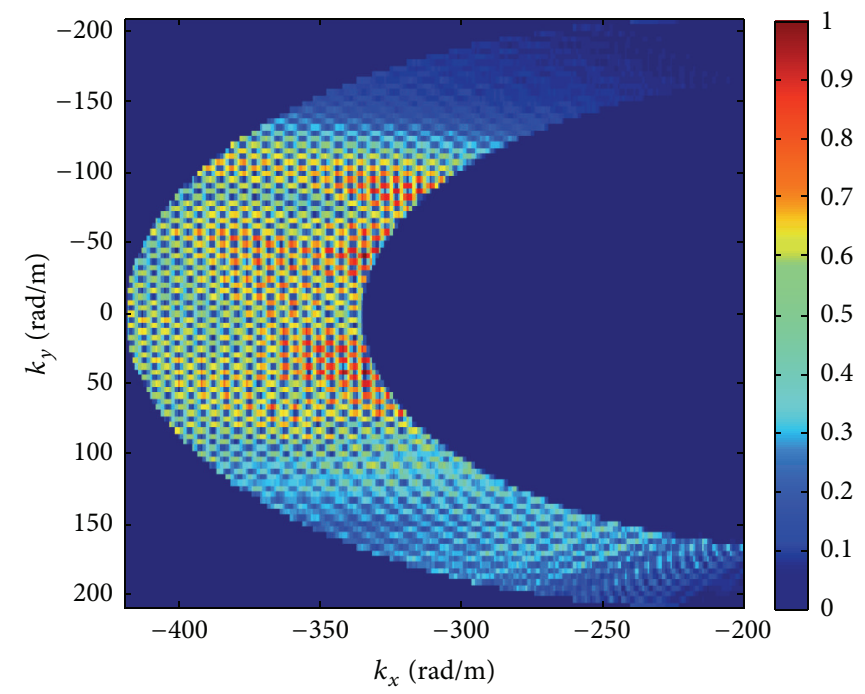

(a)

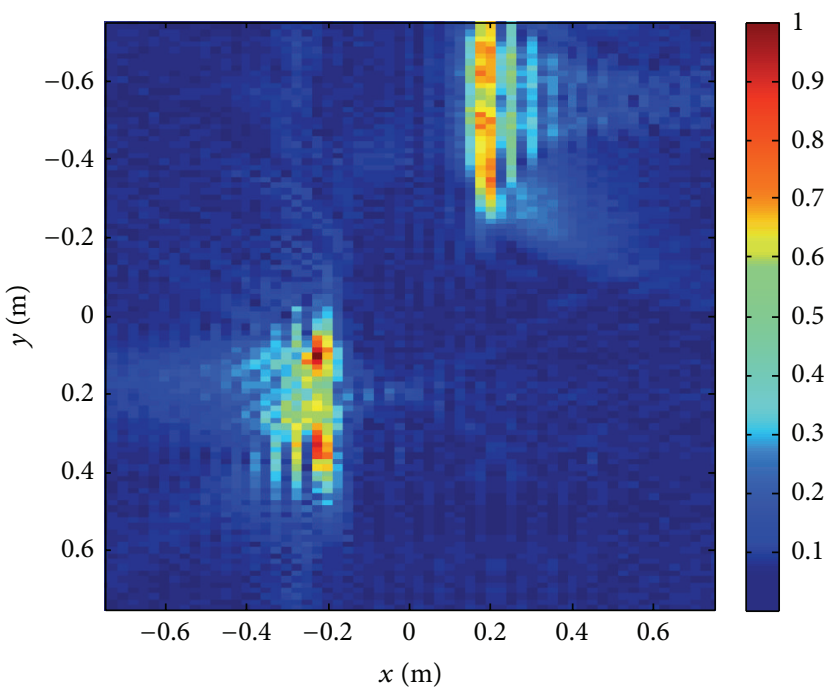

(b)

FIGURE 20: (a) The magnitude of Fourier space data based on the transmission of the first derivative Gaussian waveform shown in Figure 17(a) for two randomly distributed cylindrical PEC objects shown in Figure 13. (b) The tomographic image of two randomly distributed cylindrical conducting objects located at $(-7.5 \mathrm{~cm}, 22.5 \mathrm{~cm})$ and $(35 \mathrm{~cm},-55 \mathrm{~cm})$.

due to the multiple PEC objects are also imaged in all tomographic images.

\subsection{Diffraction Tomography with Multiple Transmitted iid} WGN Waveforms for Two Randomly Distributed Scattering Objects. Two-dimensional backward scattering geometry for two randomly distributed cylindrical conducting objects is shown in Figure 13. The radii of both cylinders are $15 \mathrm{~cm}$, and the positions of two cylinders are $(-7.5 \mathrm{~cm}$, $22.5 \mathrm{~cm})$ and $(35 \mathrm{~cm},-55 \mathrm{~cm})$. The general configuration of simulation geometry, such as number of transmitted UWB
WGN waveforms, frequency swept ranges, coordinates of the linear receiving array, and the receiver spacing, is identical to the previous cases.

Four final tomographic images for two randomly distributed PEC cylinders are displayed in Figures 14(a), 14(b), 14(c), and 14(d) when one, three, seven and all ten discrete images are summed and averaged, respectively. Again, the mutual coupling effects are shown in the tomographic images as expected. The reconstructed images are shown to be in good agreement with the simulation geometry given in Figure 13. 


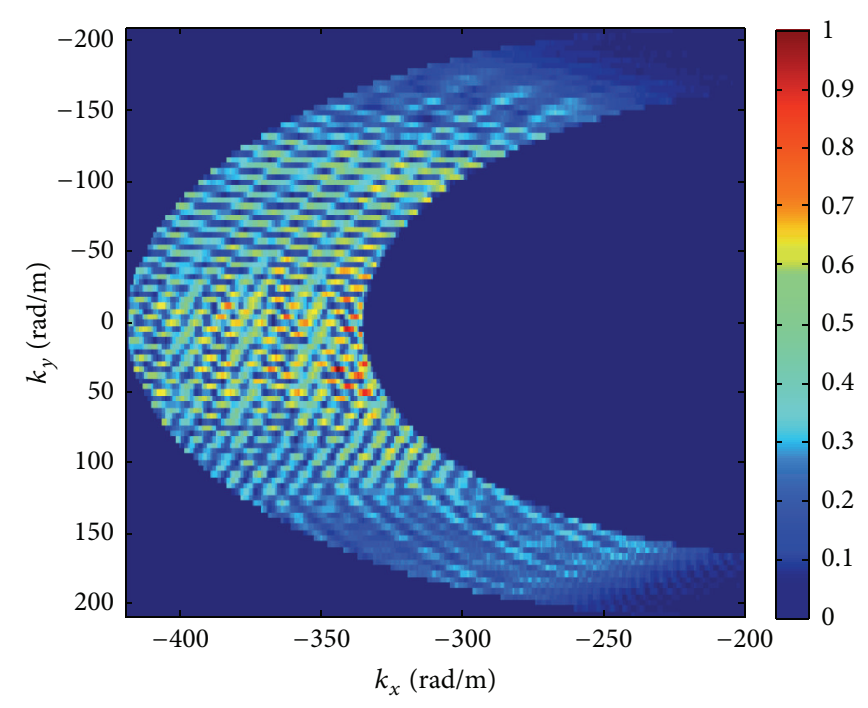

(a)

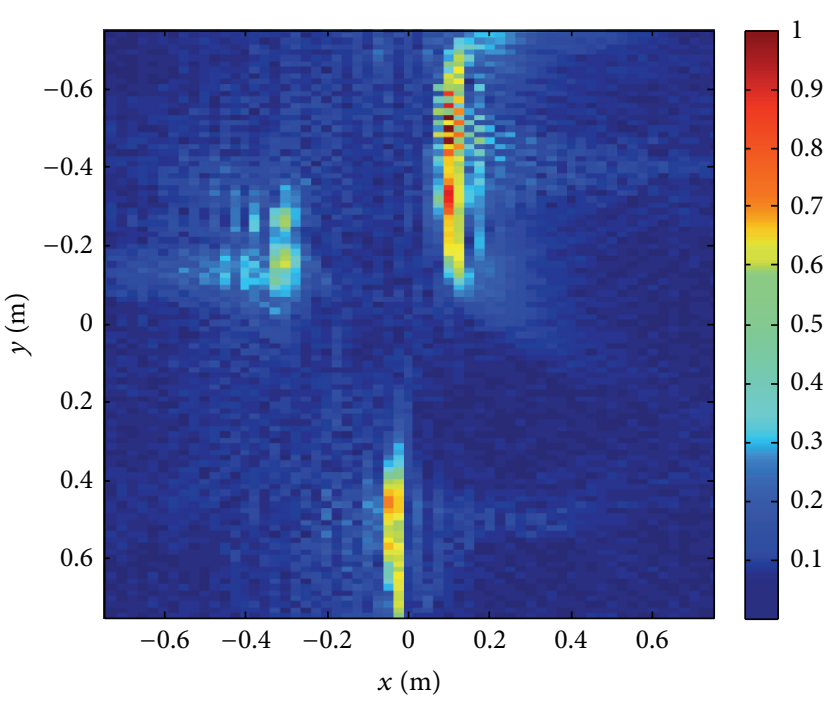

(b)

FiguRE 21: (a) The magnitude of Fourier space data based on the transmission of the first derivative Gaussian waveform shown in Figure 17(a) for three randomly distributed cylindrical PEC objects in different sizes shown in Figure 15. (b) The tomographic image of three randomly distributed cylindrical conducting objects in different sizes located at $(-22.5 \mathrm{~cm},-20 \mathrm{~cm}),(10 \mathrm{~cm}, 50 \mathrm{~cm})$, and $(25 \mathrm{~cm},-40 \mathrm{~cm})$.

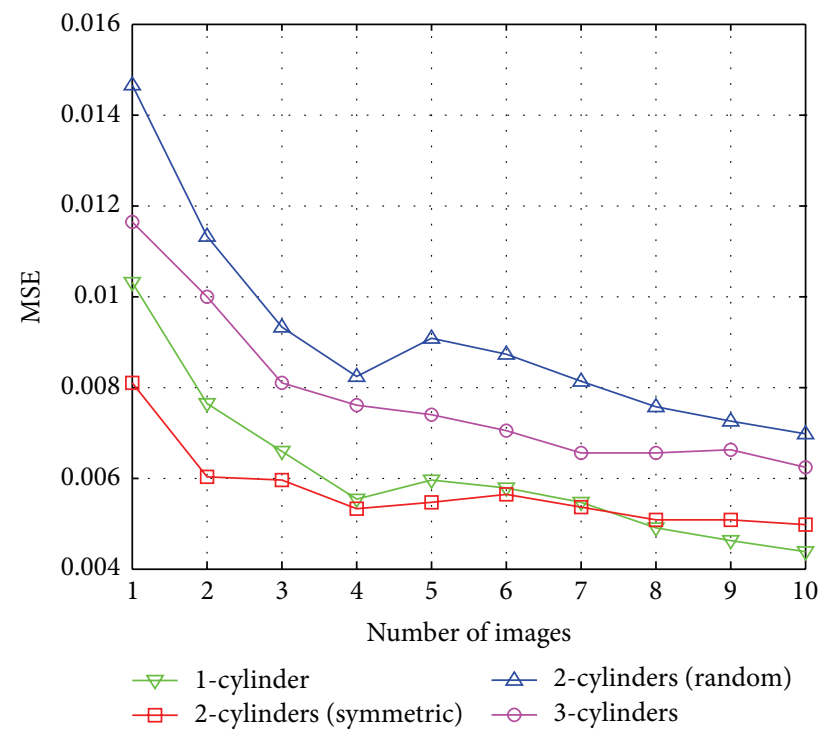

FIGURE 22: MSE versus number of reconstructed images after normalization. MSE decreases as the number of reconstructed images is overlapped.

4.5. Diffraction Tomography with Multiple Transmitted iid WGN Waveforms for Three Randomly Distributed Scattering Objects in Different Sizes. As shown in Figure 15, three PEC cylinders with radii of $7.5 \mathrm{~cm}, 10 \mathrm{~cm}$, and $15 \mathrm{~cm}$ are located at $(-22.5 \mathrm{~cm},-20 \mathrm{~cm}),(10 \mathrm{~cm}, 50 \mathrm{~cm})$, and $(25 \mathrm{~cm},-40 \mathrm{~cm})$, respectively. Again, the general configurations of simulation geometry remain unchanged as defined in previous cases.

Figures 16(a), 16(b), 16(c), and 16(d) show four final tomographic images for the scattering geometry given in Figure 15 when one, three, seven and all ten discrete images are summed and averaged, respectively. As shown in the previous cases, the image quality of reconstructed tomographic images for unevenly distributed multiple scattering objects is affected by the mutual coupling effects. Successful tomographic image of the target is reconstructed based on multiple transmitted noise waveforms as shown in Figure 16(d).

Based on the numerical simulation results of various scattering target geometries using multiple band-limited iid UWB WGN waveforms, we conclude that increasing the number of transmissions of the iid UWB WGN waveform 


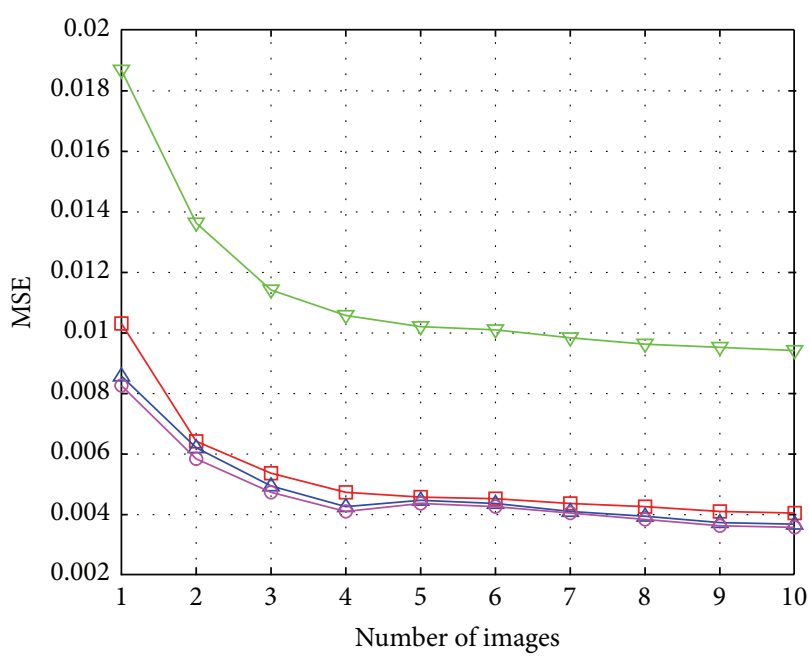

(a)

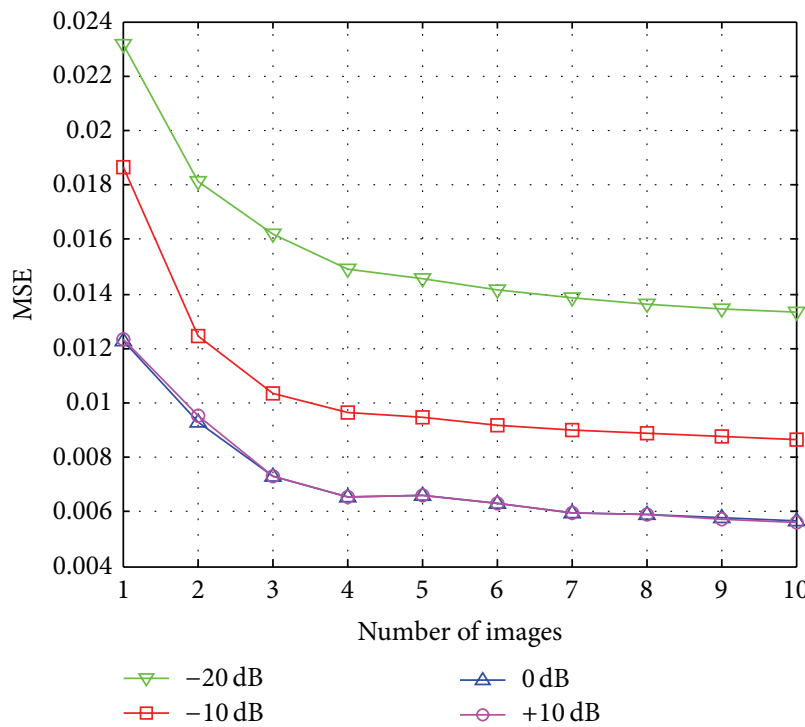

(c)

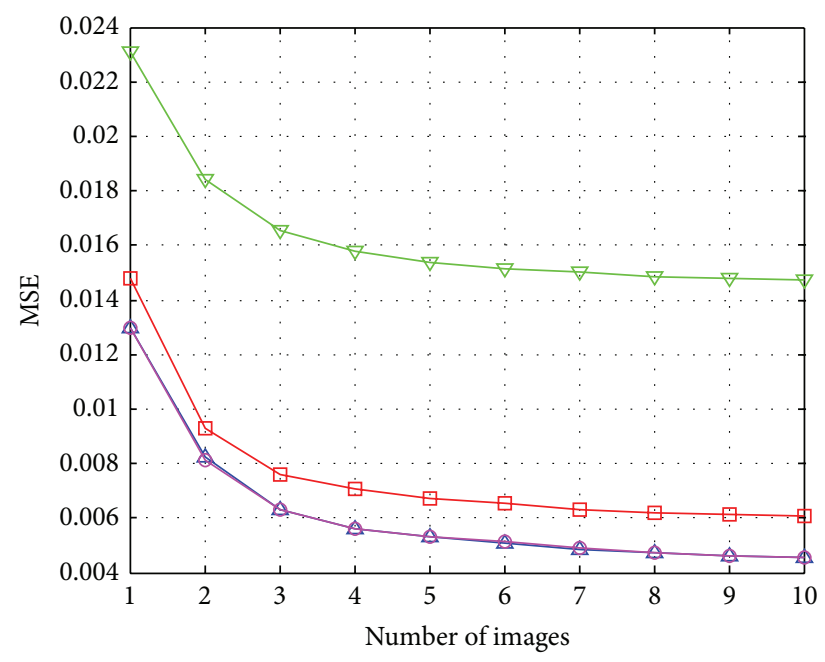

(b)

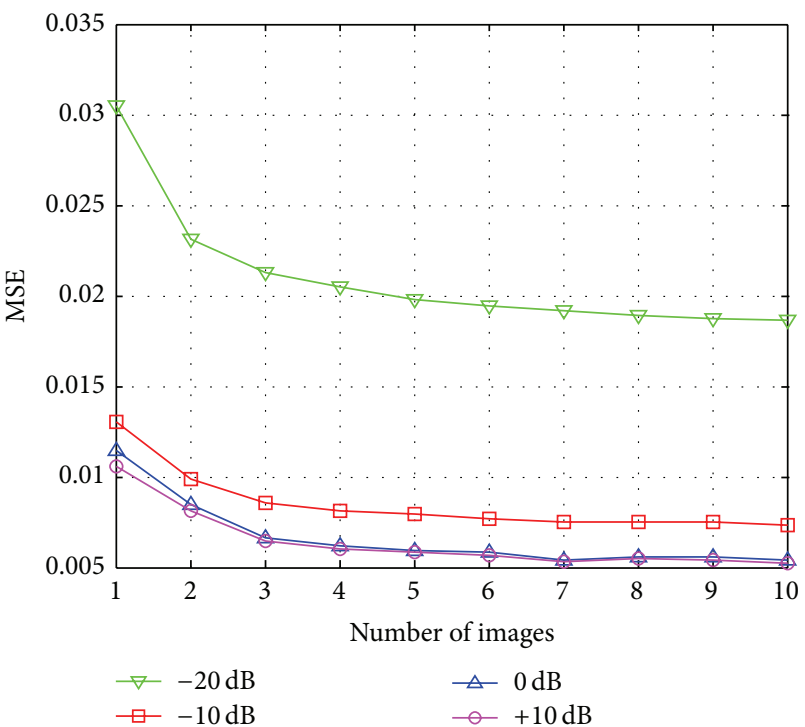

(d)

FIGURE 23: MSE versus number of reconstructed images after normalization with 4 different SNR values for (a) single cylindrical PEC object, (b) two symmetrically distributed cylindrical PEC objects, (c) two randomly distributed cylindrical PEC objects, and (d) three randomly distributed cylindrical PEC objects in different sizes. For all cases, MSE decreases as the number of reconstructed images is overlapped regardless of SNRs.

improves the quality of tomographic image by reducing the variance of the spectral response of WGN as stated in Section 2.

\section{Image Quality Measure}

As concluded in Section 4, increasing the number of transmissions of the iid WGN waveform tends to enhance the formed tomographic image. The image quality measures (IQMs) are discussed to determine the quality of tomographic images based on quantitative analysis. A good subjective assessment is required to evaluate the image quality and the performance of imaging systems. Various methods to measure the image quality and investigate their statistical performance have been studied [34]. The most frequently used measures are deviations between the reference and reconstructed image with varieties of the mean square error (MSE) $[35,36]$. The reasons for the widespread popularity of the analysis based on MSE calculations are their mathematical tractability and the fact that it is often straightforward to design systems that minimize the MSE.

5.1. Reference Image Generation. Prior to measuring the image quality of reconstructed tomographic images shown in the previous section, a "reference" image is obtained via diffraction tomography proposed in Figure 6 using the first 


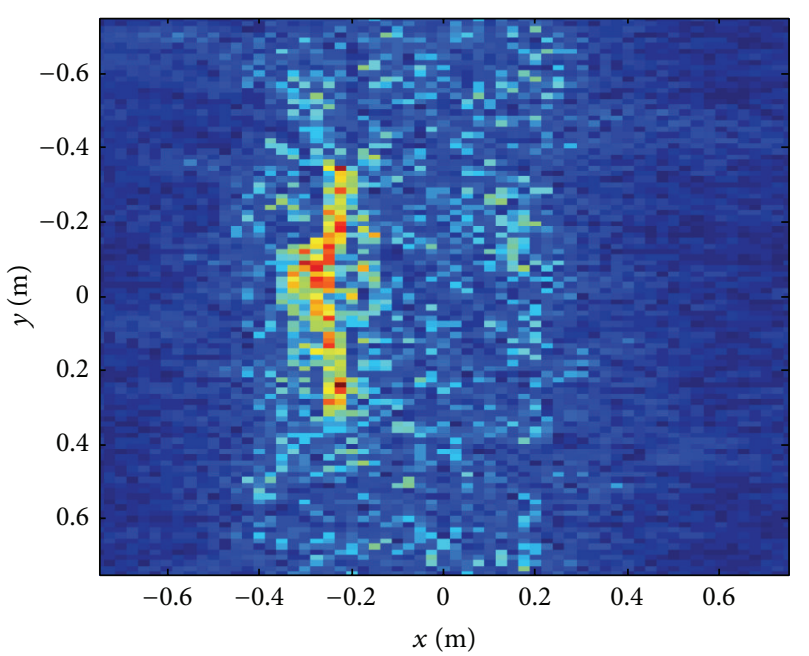

(a)

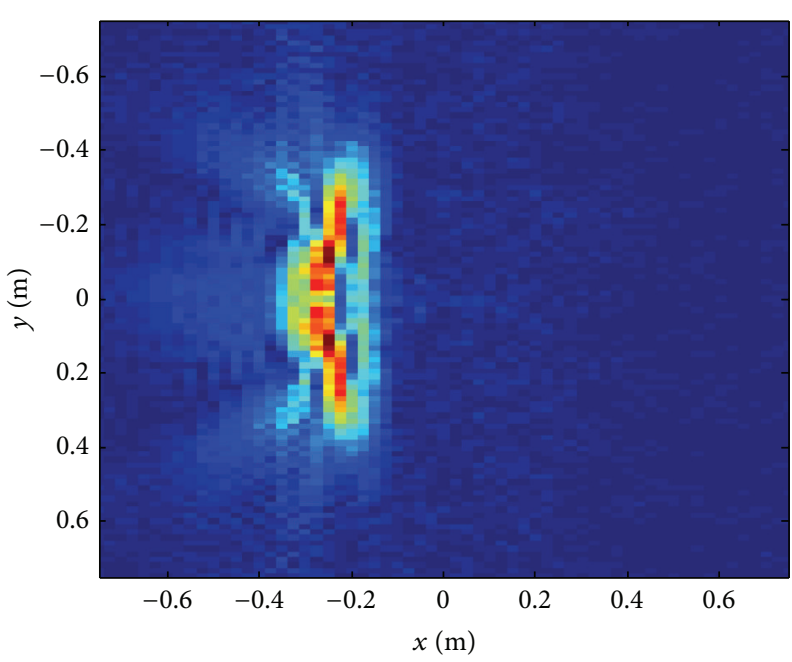

(c)

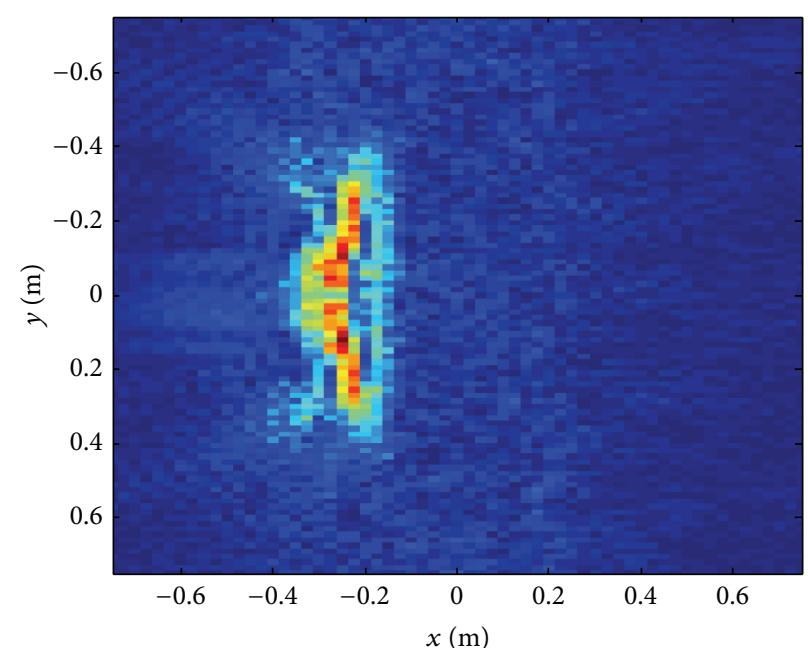

(b)

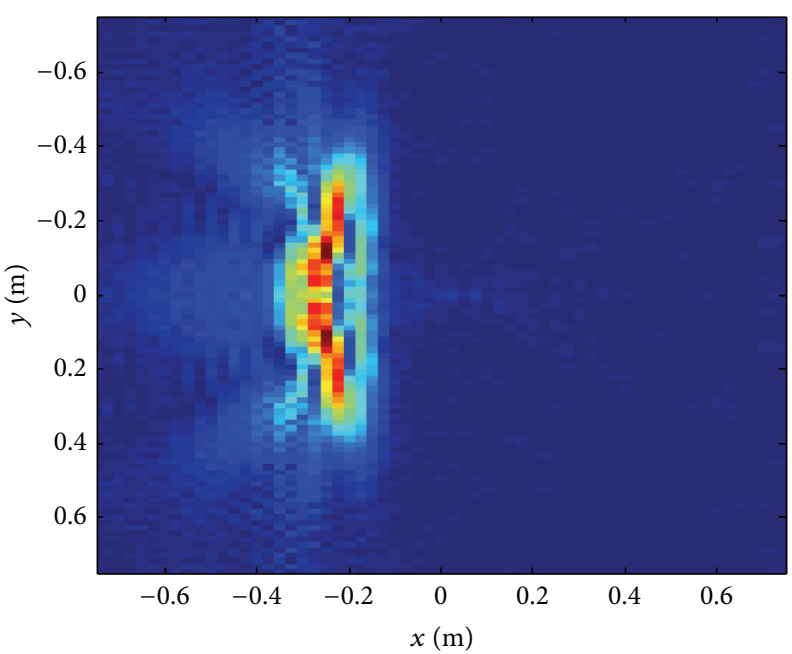

(d)

FIGURE 24: The final tomographic images after summing and averaging process with the 10 iid noise waveforms with various SNRs for a single PEC cylinder: (a) SNR $=-20 \mathrm{~dB}$, (b) SNR $=-10 \mathrm{~dB}$, (c) SNR $=0 \mathrm{~dB}$, and (d) SNR $=+10 \mathrm{~dB}$.

derivative Gaussian waveform. The first derivative Gaussian waveform possesses the desirable property in that it has zero average value. Since it has no DC component, the first derivative Gaussian waveform is the most popular pulse shape for various UWB applications [37, 38]; thus, it can be considered to be an appropriate waveform for generating reference images to compare with our reconstructed images. Figure 17(a) displays the first derivative Gaussian input waveform with a pulse width of $0.1 \mathrm{~ns}$, and the frequency spectrum of the pulse is relatively flat in the range of $8-10 \mathrm{GHz}$ as shown in Figure 17(b).

The Fourier space data and the tomographic image of four scattering target geometries in Section 4 are generated with the first derivative Gaussian input waveform shown in Figure 17(a), and they are displayed in Figures 18, 19, 20, and 21 . These tomographic images are considered to be the reference images for IQM analysis.
5.2. Pixel Difference-Based Measure. The tomographic images shown in Section 4 are obtained based on 10 iid UWB WGN transmitted waveforms, and the image enhancement technique is not implemented in diffraction tomography algorithm. Every pixel in all images is generated based on the scattering object function defined in (22). MSE is the cumulative mean squared error between the corresponding pixels of the reference images and the reconstructed images based on multiple iid UWB WGN transmitted waveforms. MSE is defined as

$$
\mathrm{MSE}=\frac{1}{M N} \sum_{m=0}^{M-1} \sum_{n=0}^{N-1}\left[R\left(x_{m}, y_{n}\right)-S\left(x_{m}, y_{n}\right)\right]^{2}
$$

where $R\left(x_{m}, y_{n}\right)$ and $S\left(x_{m}, y_{n}\right)$ represent the value of each pixel of the reference image and reconstructed tomographic 


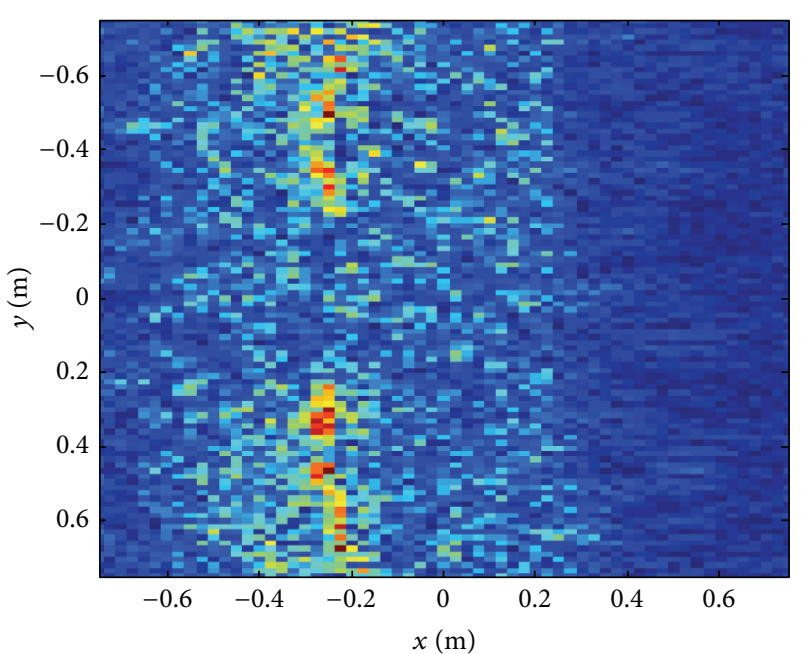

(a)

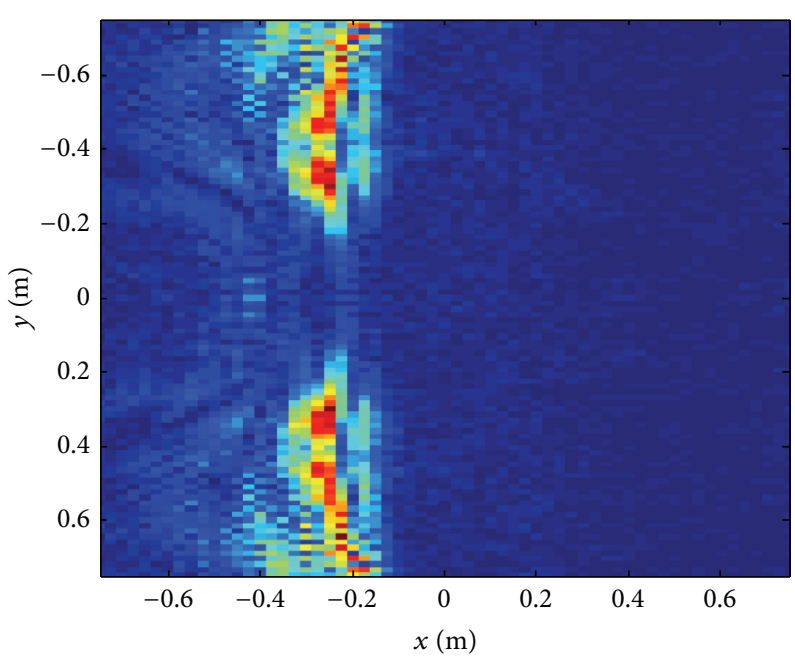

(c)

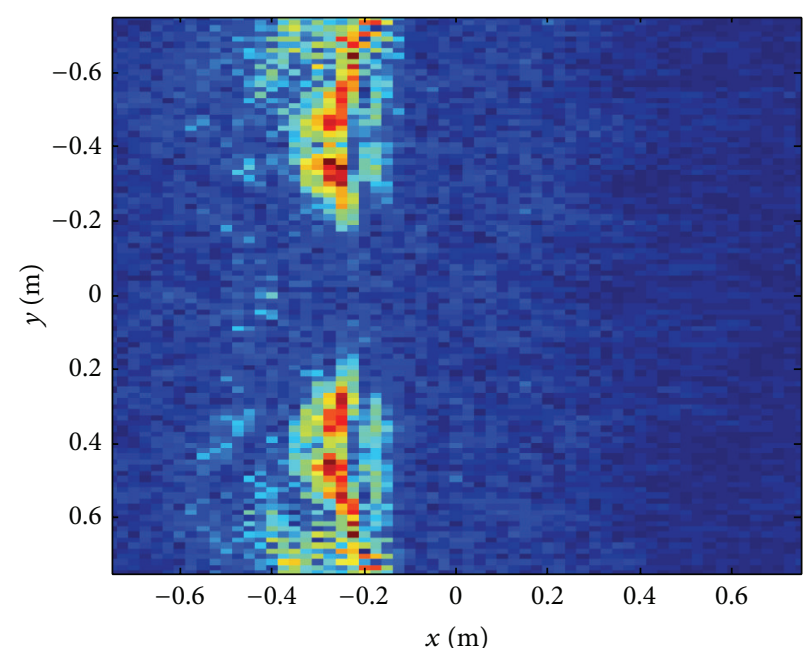

(b)

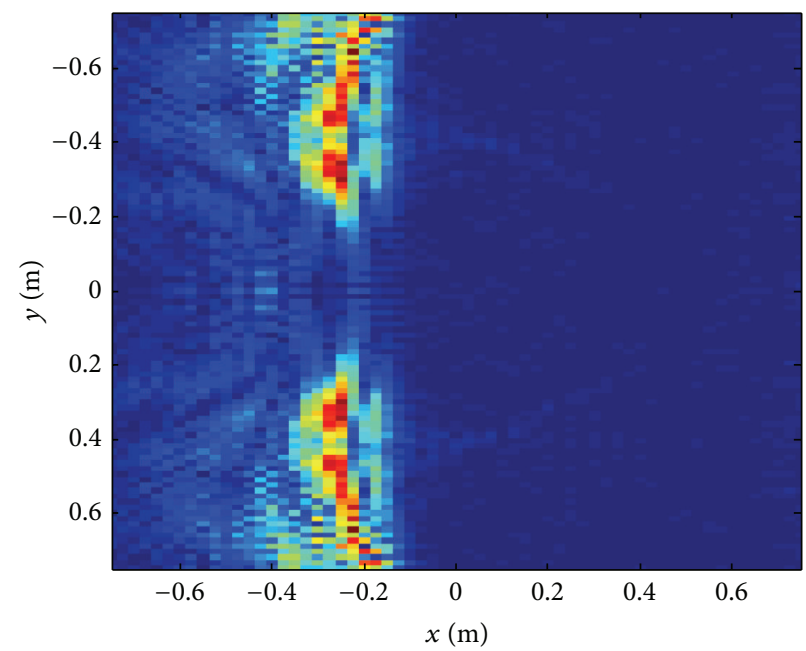

(d)

FIGURE 25: The final tomographic images after summing and averaging process with the 10 iid noise waveforms with various SNRs for two symmetrically distributed PEC cylinders: (a) SNR $=-20 \mathrm{~dB}$, (b) SNR $=-10 \mathrm{~dB}$, (c) $\mathrm{SNR}=0 \mathrm{~dB}$, and (d) $\mathrm{SNR}=+10 \mathrm{~dB}$.

images with multiple iid UWB WGN transmitted waveforms, respectively, and $M$ and $N$ are the dimensions of the images.

Each pixel of tomographic images represents the normalized magnitude of scattering object function, $o(x, y)$, and the dimensions of images are equivalent to the number of pixels across the $x$-axis and $y$-axis. By using (24), MSE values are calculated to evaluate the deviation of the pixel values from those of the corresponding reference image, as the iid noise images are summed, averaged, and normalized. Figure 22 displays the MSE versus number of overlapping reconstructed images. Such quantitative analysis shows that the tomographic images of the objects become clear and distinctive as the number of reconstructed images based on iid WGN waveforms increases. To be more specific, as shown in Figure 22, MSE decreases as the number of overlapped reconstructed images based on iid UWB WGN transmitted waveforms increases.
5.3. Signal-to-Noise Ratio Effects on Image Quality. All tomographic images shown above are formed in the absence of noise in the system; that is, signal-to-noise ratio (SNR) is equal to infinity. In practical situations, the received signal shows unpredictable perturbations due to the contributions of various noise sources in communication and imaging systems $[39,40]$. For example, thermal noise is present in all electronic devices and transmission media and is uniformly distributed across the frequency spectrum. When backscattering data acquisition is performed with a signal analyzer, the recorded data consists of scattered field information with some thermal noise from the equipment and cables. However, the collected data can be viewed as the sum of the scattered field and the additive white Gaussian noise since they are uncorrelated.

In this section, the white Gaussian noise is added accordingly to the collected scattered field dataset, establishing 


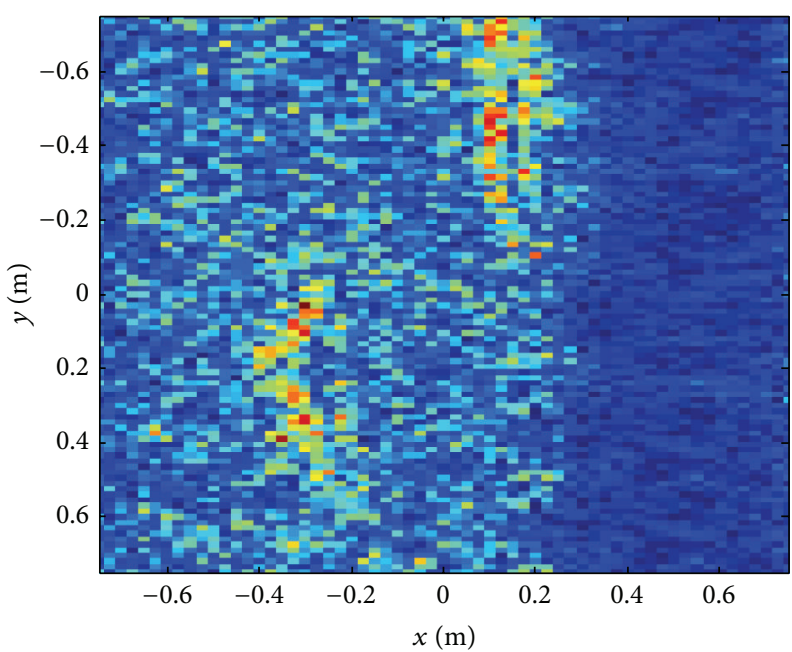

(a)

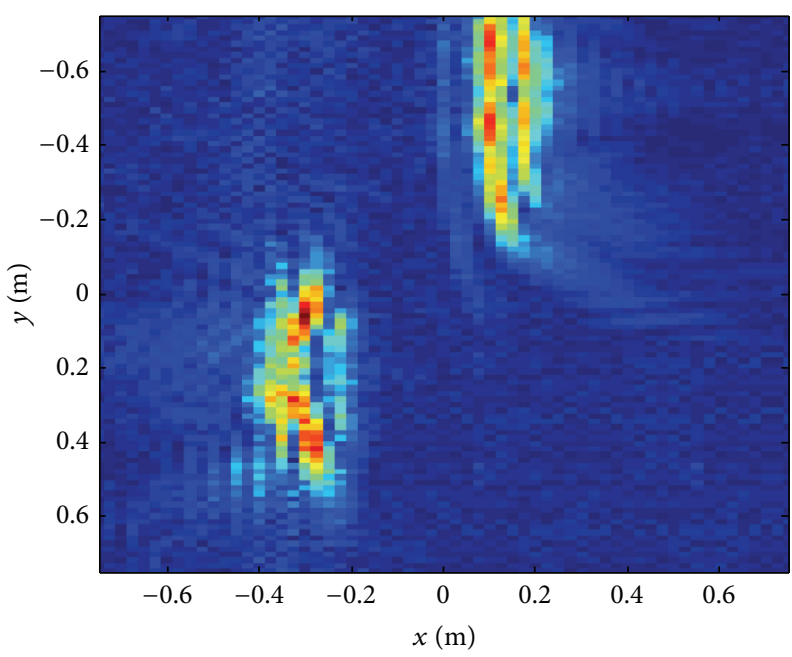

(c)

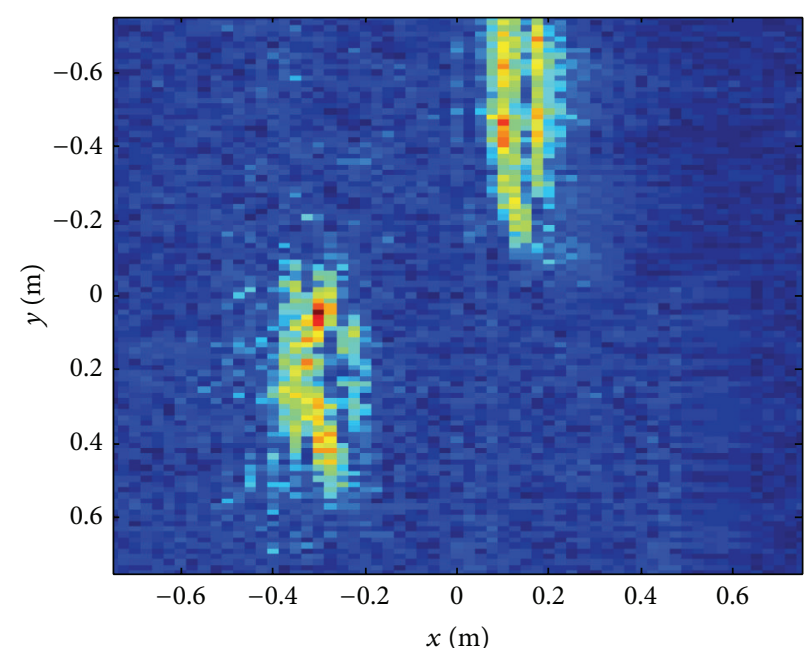

(b)

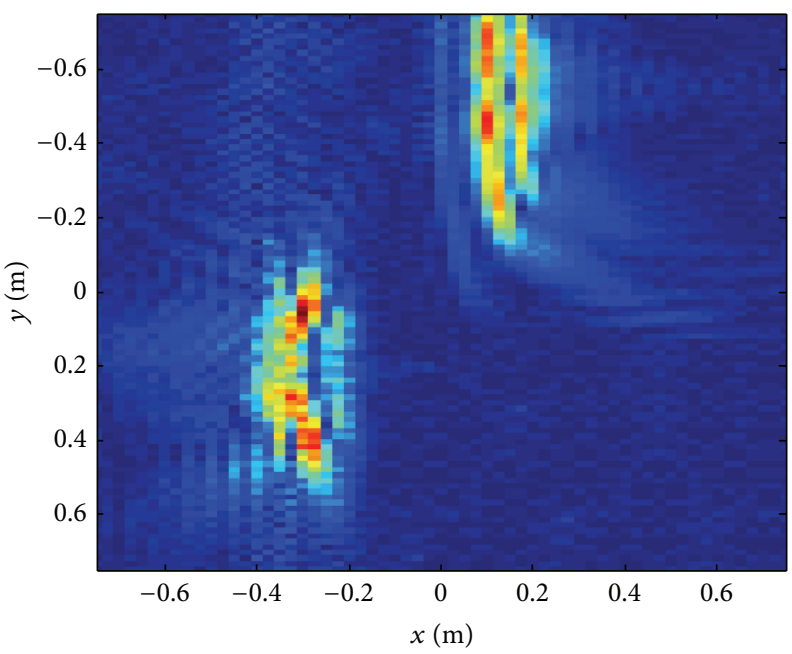

(d)

FIGURE 26: The final tomographic images after summing and averaging process with the 10 iid noise waveforms with various SNRs for two randomly distributed PEC cylinders: (a) SNR $=-20 \mathrm{~dB}$, (b) SNR $=-10 \mathrm{~dB}$, (c) SNR $=0 \mathrm{~dB}$, and (d) $\mathrm{SNR}=+10 \mathrm{~dB}$.

simulation environment with 4 different SNR values: $-20 \mathrm{~dB}$, $-10 \mathrm{~dB}, 0 \mathrm{~dB}$, and $+10 \mathrm{~dB}$. Prior to measuring the quality of the final tomographic images with various SNRs, MSE values are calculated to determine the deviation of the pixel values from that of the corresponding reference image, as the iid noise images generated with the corresponding SNR values are summed, averaged, and normalized. Based on the previous quantitative analysis results shown in Figure 22, the image quality of the tomographic image is expected to be enhanced as the number of reconstructed images increases regardless of SNR values. Figure 23 displays the MSE versus number of overlapping reconstructed images with 4 different SNRs for four scattering target geometries shown in Section 4.

After completion of total 10 transmissions of iid noise waveforms for 4 different SNR values defined above, the final tomographic images of all four scattering geometries are achieved and displayed in Figures 24, 25, 26, and 27.
As shown in Figure 24 through Figure 27, the image quality of the final tomographic image after summing, averaging, and normalizing process with transmitting 10 iid noise waveforms is truly affected by the additive white Gaussian noise. By visual inspection of the formed images, the image degradation due to the additive Gaussian noise is clearly displayed when SNR is set to $-20 \mathrm{~dB}$. In contrast, no such image degradation is observed at relatively higher SNRs, that is, SNR of $+10 \mathrm{~dB}$.

In order to evaluate the overall impact of noise effect on the formed tomographic images, MSE is calculated at the SNR range from $-20 \mathrm{~dB}$ to $+10 \mathrm{~dB}$. Figure 28 explains the relationship between the image quality degradation and SNR. The rate of change in MSE is the maximum at the SNR range between $-20 \mathrm{~dB}$ and $-10 \mathrm{~dB}$, and this is the SNR range where the final tomographic image is severely degraded by unwanted noise. However, the rate of change 


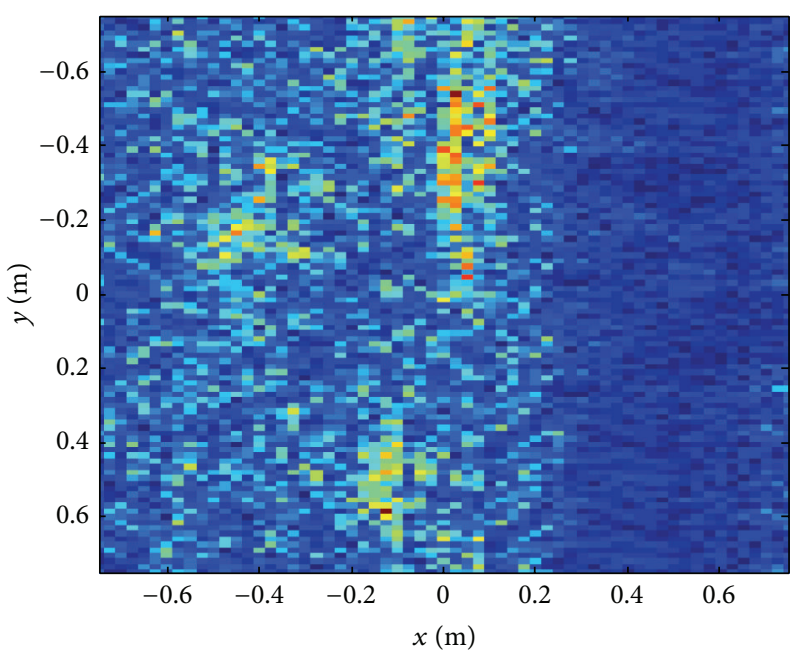

(a)

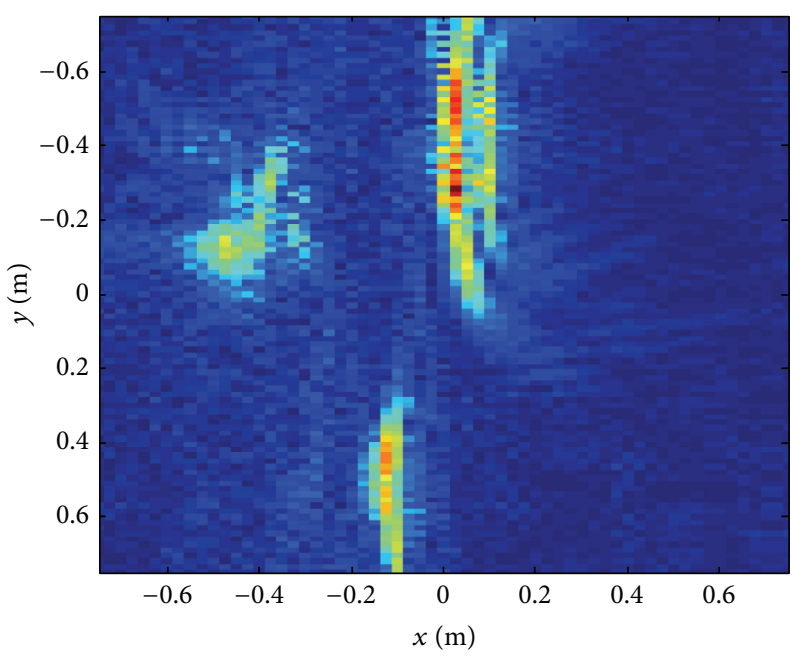

(c)

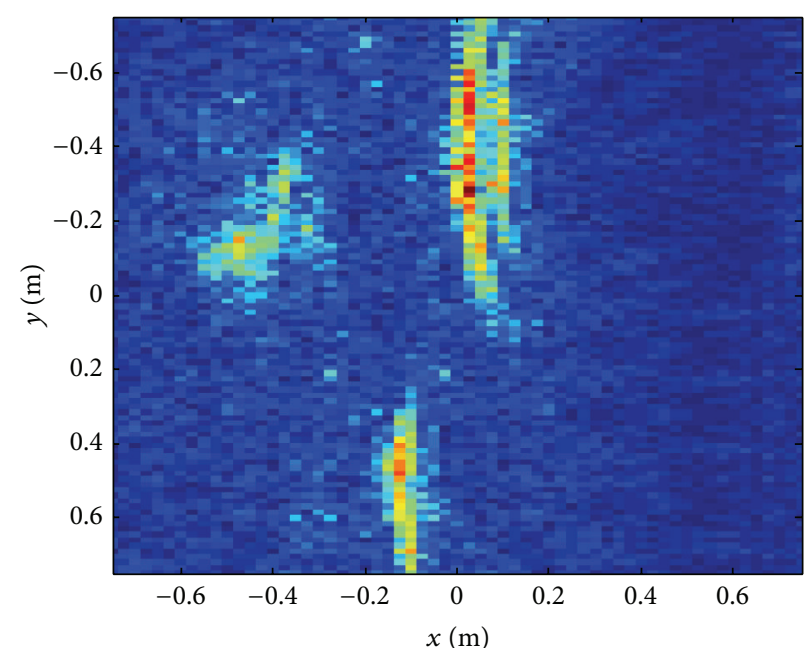

(b)

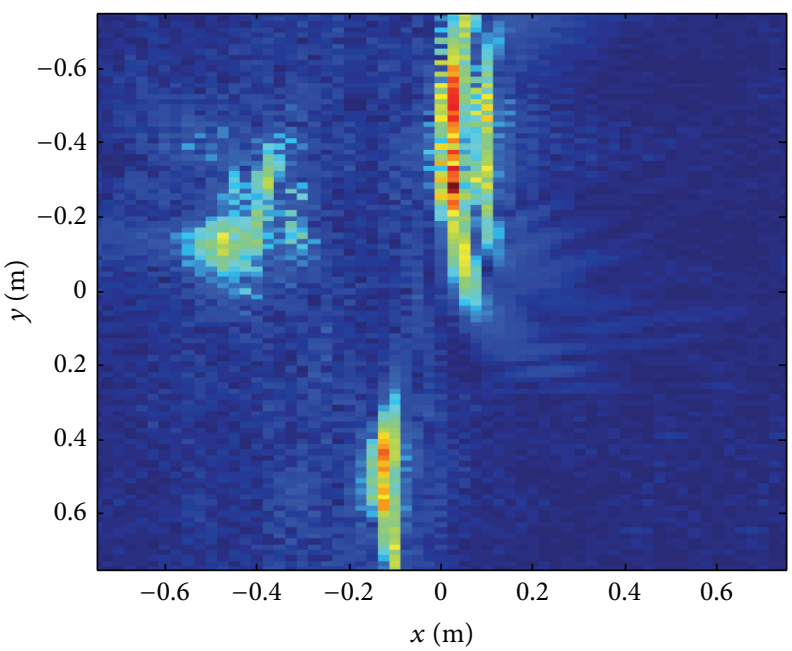

(d)

FIGURE 27: The final tomographic images after summing and averaging process with the 10 iid noise waveforms with various SNRs for three randomly distributed PEC cylinders in different sizes: (a) SNR $=-20 \mathrm{~dB}$, (b) SNR $=-10 \mathrm{~dB}$, (c) $\mathrm{SNR}=0 \mathrm{~dB}$, and (d) SNR $=+10 \mathrm{~dB}$.

in MSE starts falling as SNR increases, which indicates that the tomographic image is less affected by the noise contributions at high SNR. The results shown in Figure 28 are the rough measure to estimate the image quality on the final tomographic images in practical implementation of the imaging system and designs for actual experiment.

5.4. Preliminary Experimental Validation. The two-dimensional backward scattering geometry with a single cylindrical conducting object shown in Figure 3 is established for validating simulation results. The basic hardware configuration for the preliminary experiment is shown in Figure 29. For data acquisition, a scanner is configured to transmit the noise waveform via $\mathrm{Tx}$ antenna and collect the scattered field via $\mathrm{Rx}$ antenna at the same location on the $y$-axis, and both $\mathrm{Tx}$ and $\mathrm{Rx}$ antennas move along the scanning direction after collecting scattering data. The data acquisition process repeats for 101 positions along the scanning direction. The first and last scanning positions are $(-89 \mathrm{~cm}$, $-40 \mathrm{~cm}$ ) and $(-89 \mathrm{~cm}, 40 \mathrm{~cm})$, respectively. In the transmit chain, the arbitrary waveform generator (AWG) generates a white Gaussian noise waveform with 500 random amplitude samples over the $2 \mathrm{GHz}$ to $4 \mathrm{GHz}$ frequency range, and a low pass filter (LPF) for frequencies from DC to $4 \mathrm{GHz}$ is implemented to reject undesired high frequency signals coming from the AWG before frequency mixing. A signal generator provides the $6 \mathrm{GHz}$ signal for upconversion, and a mixer is used to upconvert the generated noise waveform frequency from $8 \mathrm{GHz}$ to $10 \mathrm{GHz}$. The receiver is designed to collect the scattering data with a signal analyzer, and the final tomographic image of the target is obtained after postprocessing with a computer.

For validation purposes, only a single noise waveform is transmitted to validate the simulation results by generating the tomographic image of the target. Experimental results of Fourier space data and the tomographic images 


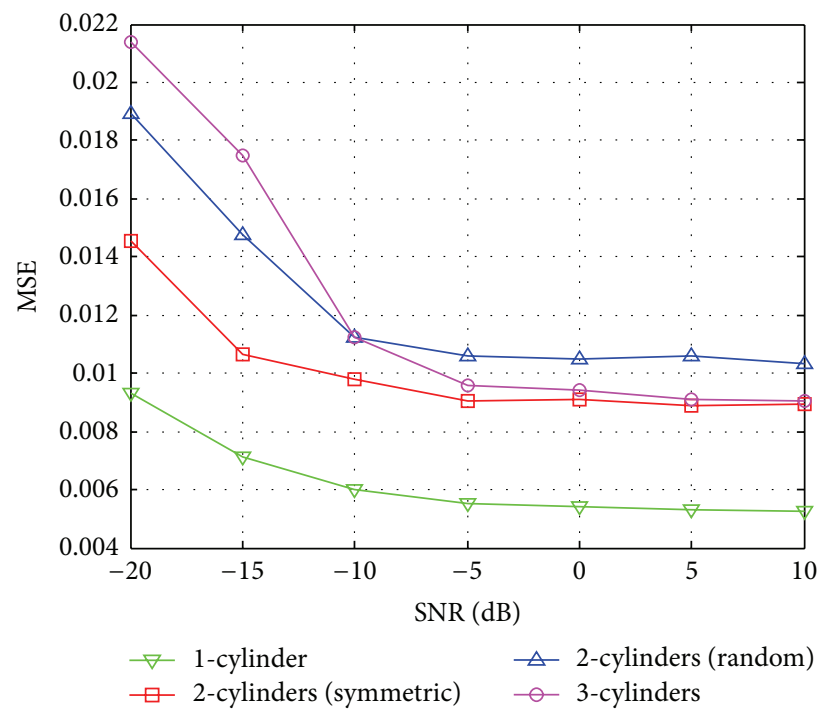

FIGURE 28: MSE versus SNR values for all final tomographic images shown in Figure 24 through Figure 27. MSE decreases as the level of SNR increases.

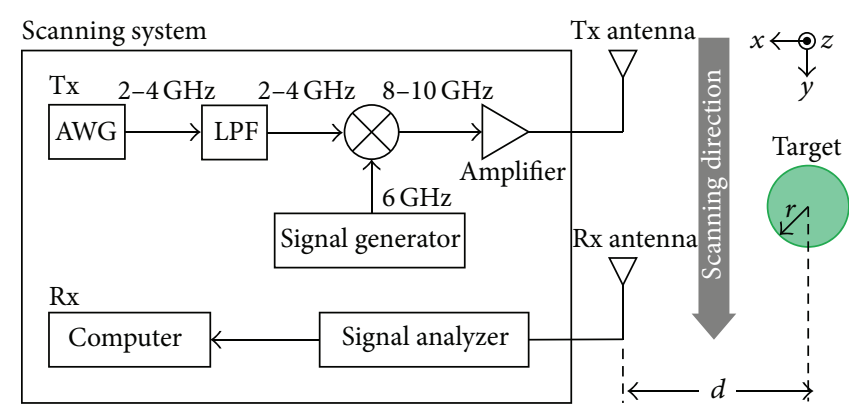

(a)

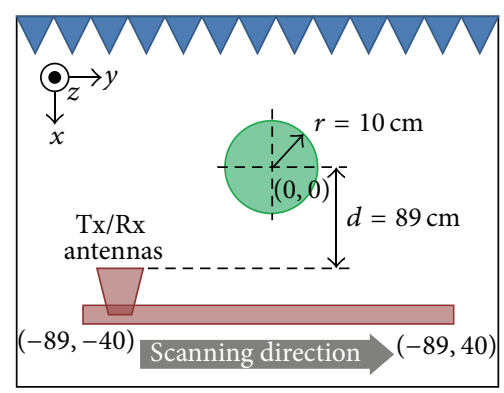

(b)

Figure 29: (a) Experiment setup for the two-dimensional backward scattering. The scanner is designed to transmit and receive the signals along the scanning direction. (b) Top view of experiment configuration for a single scattering geometry. The radius $(r)$ of the cylindrical target is $10 \mathrm{~cm}$, and the distance $(d)$ from the target to the antennas is $89 \mathrm{~cm}$.

of the single cylindrical conducting object are displayed in Figures 30(a) and 30(b), respectively. As shown in Figure 30, the experimental results compare well with the numerical simulation results shown in Figures 7 and 8. A more detailed experimental configuration and comprehensive results for various scattering geometries with multiple transmissions of iid noise waveforms will be presented and published soon.

\section{Conclusion}

This paper shows that tomographic images of scattering objects are successfully achieved with multiple transmissions of random noise waveforms. From the simulation results, we conclude that a single transmitted UWB WGN may not be sufficient to generate the correct tomographic image due to the practical implementation of a transmitted random noise waveform. However, multiple iid UWB WGN transmitted waveforms can bypass the shortcoming of a single transmission of UWB WGN waveform, forming a correct image of target objects by summing and averaging discrete object images based on each iid WGN waveform.

Also the image quality of the tomographic image after completion of multiple transmissions of iid noise waveforms is analyzed. MSE is used to measure the image quality of all tomographic images in this paper. Image quality of the tomographic images based on the random noise waveform is enhanced as the number of iid noise waveform transmissions increases. The presence of white Gaussian noise degrades the image quality; however, the suppression of unwanted noise contributions by controlling SNR can help to achieve successful tomographic images in practical radar imaging systems. Also, numerical simulation results for the single scattering object scenario are validated with the preliminary experiment results. 


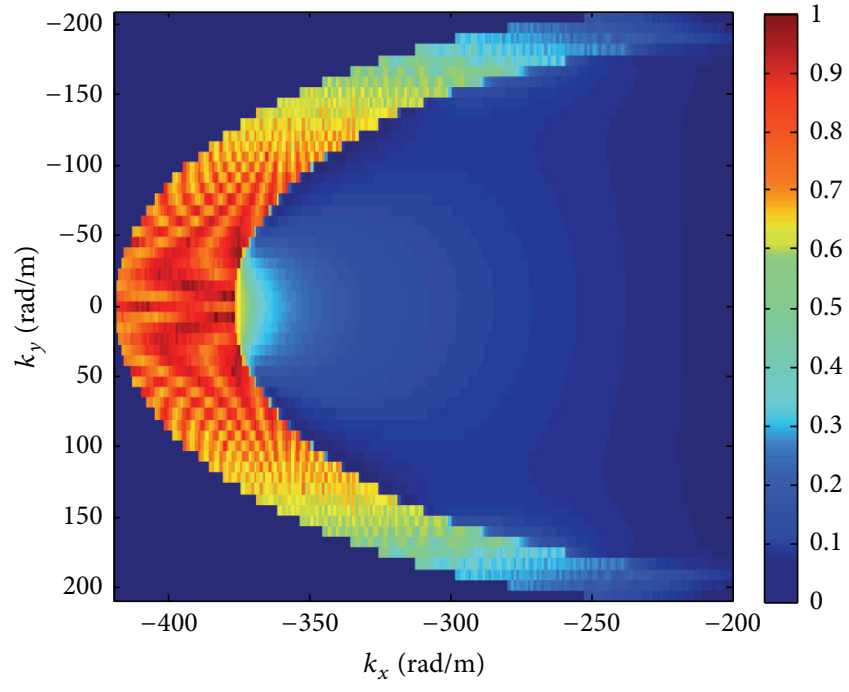

(a)

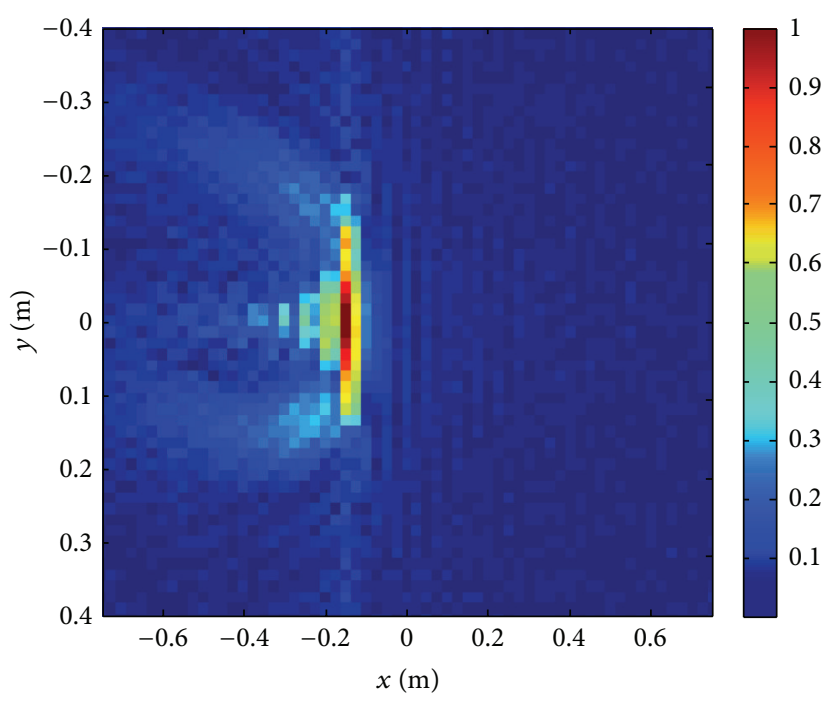

(b)

FIGURE 30: Experimental results of the single cylindrical conducting object geometry shown in Figure 3. (a) The magnitude of Fourier space data of the single cylindrical conducting object. The colorbar indicates the normalized magnitude of $\widetilde{O}\left(k_{x}, k_{y}\right)$. (b) The obtained tomographic image after transmitting a single UWB WGN waveform. The colorbar indicates the normalized magnitude of scattering object function, $o(x, y)$.

\section{Conflict of Interests}

The authors declare that there is no conflict of interests regarding the publication of this paper.

\section{Acknowledgment}

This work was supported by the Air Force Office of Scientific Research (AFOSR) Contract no. FA9550-12-1-0164.

\section{References}

[1] B. M. Horton, "Noise-modulated distance measuring systems," Proceedings of the IRE, vol. 47, no. 5, pp. 821-828, 1959.

[2] M. P. Grant, G. R. Cooper, and A. K. Kamal, "A class of noise radar systems," Proceedings of the IEEE, vol. 51, no. 7, pp. 10601061, 1963.

[3] M. Dawood and R. M. Narayanan, "Multipath and ground clutter analysis for a UWB noise radar," IEEE Transactions on Aerospace and Electronic Systems, vol. 38, no. 3, pp. 838-853, 2002.

[4] K. Kulpa, J. Misiurewicz, Z. Gajo, and M. Malanowski, "A simple robust detection of weak target in noise radars," in Proceedings of the 4th European Radar Conference (EURAD '07), pp. 275-278, Munich, Germany, October 2007.

[5] Y. Zhang and R. M. Narayanan, "Design consideration for a real-time random-noise tracking radar," IEEE Transactions on Aerospace and Electronic Systems, vol. 40, no. 2, pp. 434-445, 2004.

[6] D. A. Ausherman, A. Kozma, J. L. Walker, H. M. Jones, and E. C. Poggio, "Developments in radar imaging," IEEE Transactions on Aerospace and Electronic Systems, vol. 20, no. 4, pp. 363-400, 1984.
[7] H. J. Shin, R. M. Narayanan, and M. Rangaswamy, "Tomographic imaging with ultra-wideband noise radar using timedomain data," in Radar Sensor Technology XVII, vol. 8714 of Proceedings of SPIE, pp. 1-9, Baltimore, Md, USA, April 2013.

[8] H. J. Shin, R. M. Narayanan, and M. Rangaswamy, "Diffraction tomography for ultra-wideband noise radar and imaging quality measure of a cylindrical perfectly conducting object," in Proceedings of the 2014 IEEE Radar Conference, pp. 702-707, Cincinnati, Ohio, USA, May 2014.

[9] L. Jofre, A. Broquetas, J. Romeu et al., "UWB tomographie radar imaging of penetrable and impenetrable objects," Proceedings of the IEEE, vol. 97, no. 2, pp. 451-464, 2009.

[10] X. Li, E. J. Bond, B. D. van Veen, and S. C. Hagness, "An overview of ultra-wideband microwave imaging via space-time beamforming for early-stage breast-cancer detection," IEEE Antennas and Propagation Magazine, vol. 47, no. 1, pp. 19-34, 2005.

[11] T. M. Grzegorczyk, P. M. Meaney, P. A. Kaufman, R. M. Diflorio-Alexander, and K. D. Paulsen, "Fast 3-D tomographic microwave imaging for breast cancer detection," IEEE Transactions on Medical Imaging, vol. 31, no. 8, pp. 1584-1592, 2012.

[12] M. H. Khalil, W. Shahzad, and J. D. Xu, "In the medical field detection of breast cancer by microwave imaging is a robust tool," in Proceedings of the 25th International Vacuum Nanoelectronics Conference (IVNC '12), pp. 228-229, Jeju Island, Republic of Korea, July 2012.

[13] Z. Wang, E. G. Lim, Y. Tang, and M. Leach, "Medical applications of microwave imaging," The Scientific World Journal, vol. 2014, Article ID 147016, 7 pages, 2014.

[14] Y. J. Kim, L. Jofre, F. De Flaviis, and M. Q. Feng, "Microwave reflection tomographic array for damage detection of civil structures," IEEE Transactions on Antennas and Propagation, vol. 51, no. 11, pp. 3022-3032, 2003.

[15] S. Kharkovsky, J. Case, M. Ghasr, R. Zoughi, S. Bae, and A. Belarbi, "Application of microwave 3D SAR imaging technique 
for evaluation of corrosion in steel rebars embedded in cementbased structures," in Review of Progress in Quantitative Nondestructive Evaluation, vol. 31, pp. 1516-1523, 2012.

[16] O. Güneş and O. Büyüköztürk, "Microwave imaging of plain and reinforced concrete for NDT using backpropagation algorithm," in Nondestructive Testing of Materials and Structures, O. Güneş and Y. Akkaya, Eds., vol. 6 of RILEM Bookseries, pp. 703709, Springer, 2013.

[17] D. Zimdars and J. S. White, “Terahertz reflection imaging for package and personnel inspection," in Terahertz for Military and Security Applications II, vol. 5411 of Proceedings of the SPIE, pp. 78-83, Orlando, Fla, USA, April 2004.

[18] S. Almazroui and W. Wang, "Microwave tomography for security applications," in Proceedings of the International Conference on Information Technology and e-Services (ICITeS '12), pp. 1-3, Sousse, Tunisia, March 2012.

[19] O. Yurduseven, "Indirect microwave holographic imaging of concealed ordnance for airport security imaging systems," Progress in Electromagnetics Research, vol. 146, pp. 7-13, 2014.

[20] L. Jofre, A. P. Toda, J. M. J. Montana et al., "UWB shortrange bifocusing tomographic imaging," IEEE Transactions on Instrumentation and Measurement, vol. 57, no. 11, pp. 2414-2420, 2008.

[21] C.-P. Lai and R. M. Narayanan, "Ultrawideband random noise radar design for through-wall surveillance," IEEE Transactions on Aerospace and Electronic Systems, vol. 46, no. 4, pp. 17161730, 2010.

[22] R. Vela, R. M. Narayanan, K. A. Gallagher, and M. Rangaswamy, "Noise radar tomography," in Proceedings of the IEEE Radar Conference: Ubiquitous Radar (RADAR '12), pp. 720-724, Atlanta, Ga, USA, May 2012.

[23] A. Leon-Garcia, Probability and Random Processes for Electrical Engineering, Addison-Wesley, Reading, Mass, USA, 2nd edition, 1994.

[24] G. Jenkins and D. Watts, Watts, Spectral Analysis and Its Applications, Holden-Day, San Francisco, Calif, USA, 1968.

[25] A. V. Oppenheim, R. W. Schafer, and J. R. Buck, Discrete-Time Signal Processing, Prentice Hall, Upper Saddle River, NJ, USA, 2nd edition, 1999.

[26] M. S. Bartlett, "Periodogram analysis and continuous spectra," Biometrika, vol. 37, pp. 1-16, 1950.

[27] S. K. Kenue and J. F. Greenleaf, "Limited angle multifrequency diffraction tomography," IEEE Transactions on Sonics and Ultrasonics, vol. 29, no. 4, pp. 213-217, 1982.

[28] B. A. Roberts and A. C. Kak, "Reflection mode diffraction tomography," Ultrasonic Imaging, vol. 7, no. 4, pp. 300-320, 1985.

[29] M. Soumekh, "Surface imaging via wave equation inversion," in Acoustical Imaging, L. W. Kessler, Ed., pp. 383-393, Springer, 1988.

[30] S. X. Pan and A. C. Kak, "A computational study of reconstruction algorithms for diffraction tomography: interpolation versus filtered-backpropagation," IEEE Transactions on Acoustics, Speech, and Signal Processing, vol. 31, no. 5, pp. 1262-1275, 1983.

[31] T.-H. Chu and K.-Y. Lee, "Wide-band microwave diffraction tomography under Born approximation," IEEE Transactions on Antennas and Propagation, vol. 37, pp. 515-519, 1992.

[32] J. C. Bolomey and C. Pichot, "Microwave tomography: from theory to practical imaging systems," International Journal of Imaging Systems and Technology, vol. 2, pp. 144-156, 1990.

[33] H. J. Shin, R. M. Narayanan, and M. Rangaswamy, "Ultrawideband noise radar imaging of cylindrical PEC objects using diffraction tomography," in Radar Sensor Technology XVIII, vol. 9077 of Proceedings of SPIE, pp. 1-10, Baltimore, Md, USA, May 2014.

[34] I. Avcibaş, B. Sankur, and K. Sayood, "Statistical evaluation of image quality measures," Journal of Electronic Imaging, vol. 11, no. 2, pp. 206-223, 2002.

[35] A. M. Eskicioglu and P. S. Fisher, "Image quality measures and their performance," IEEE Transactions on Communications, vol. 43, no. 12, pp. 2959-2965, 1995.

[36] A. M. Eskicioglu, "Application of multidimensional quality measures to reconstructed medical images," Optical Engineering, vol. 35, no. 3, pp. 778-785, 1996.

[37] J. Hu, T. Jiang, Z. Cui, and Y. Hou, "Design of UWB pulses based on Gaussian pulse," in Proceedings of the 3rd IEEE International Conference on Nano/Micro Engineered and Molecular Systems (NEMS '08), pp. 651-655, Sanya, China, January 2008.

[38] A. Thakre and A. Dhenge, "Selection of pulse for ultra wide band communication (UWB) system," International Journal of Advanced Research in Computer and Communication Engineering, vol. 1, pp. 683-686, 2012.

[39] C. E. Shannon, "Communication in the presence of noise," Proceedings of the IRE, vol. 37, pp. 10-21, 1949.

[40] M. Bertero and P. Boccacci, Introduction to Inverse Problems in Imaging, Institute of Physics Publishing, Bristol, UK, 1998. 

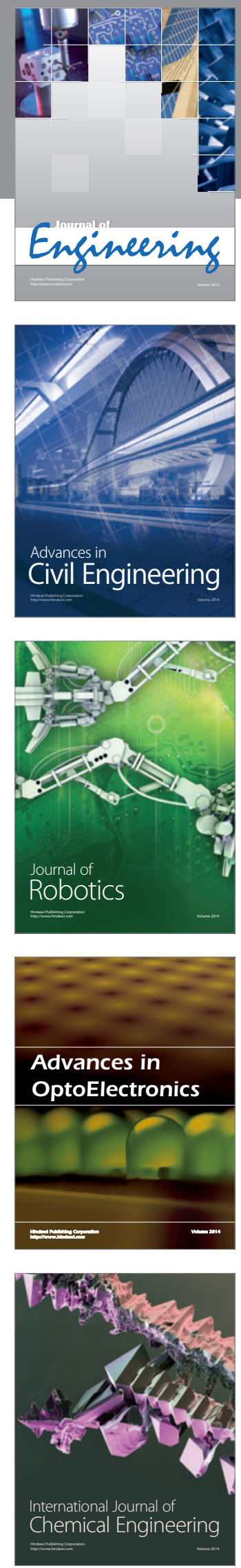

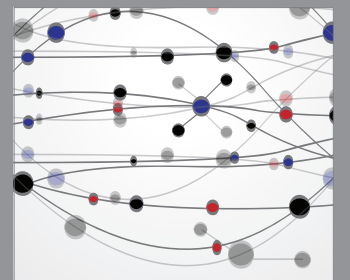

The Scientific World Journal
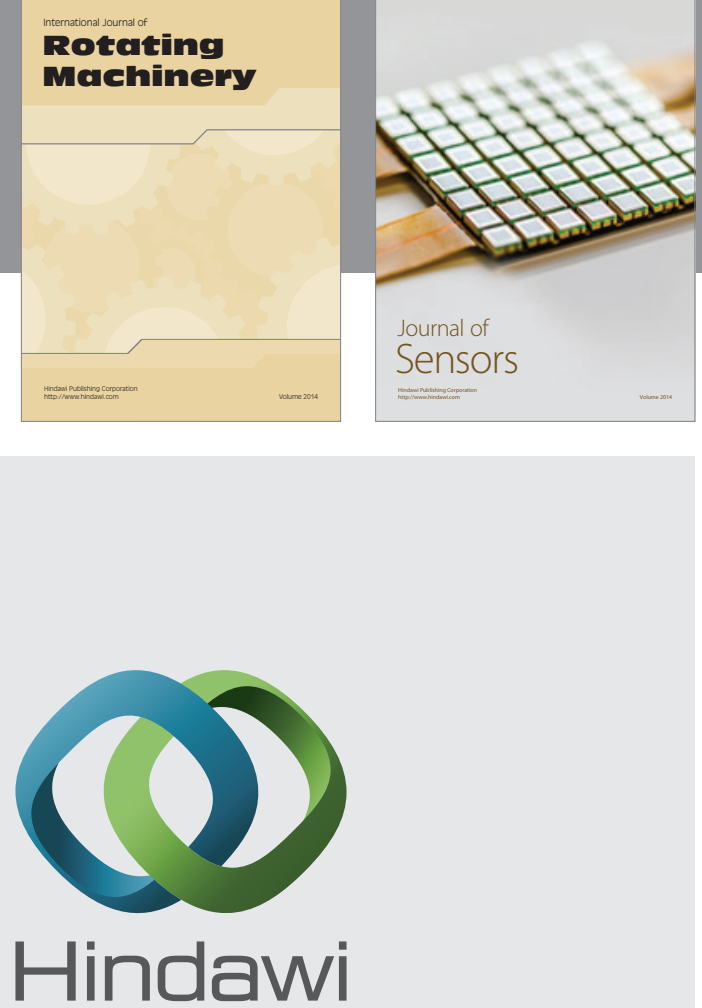

Submit your manuscripts at http://www.hindawi.com
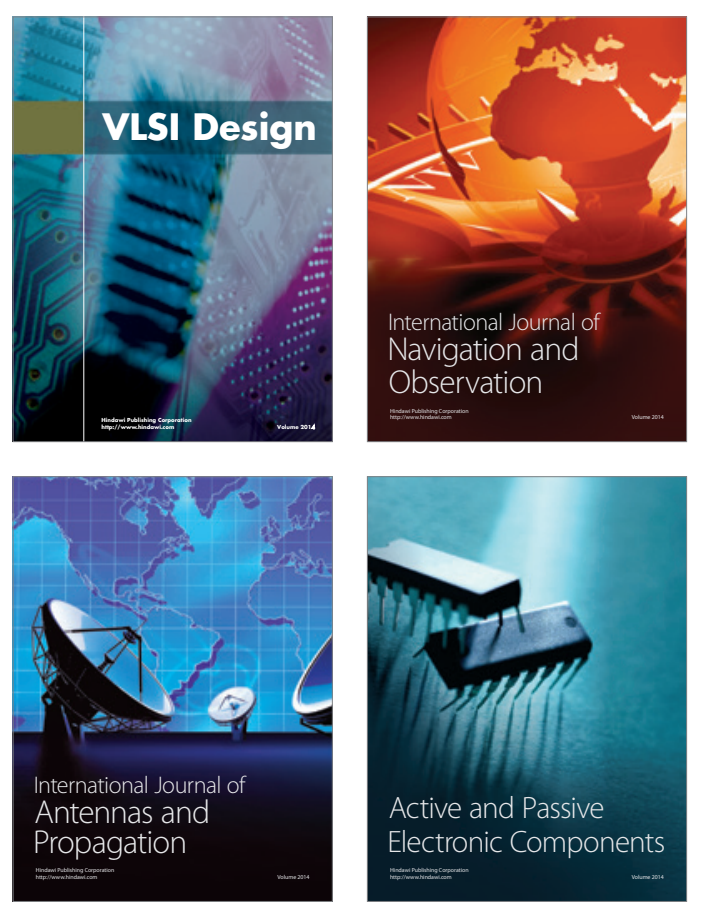
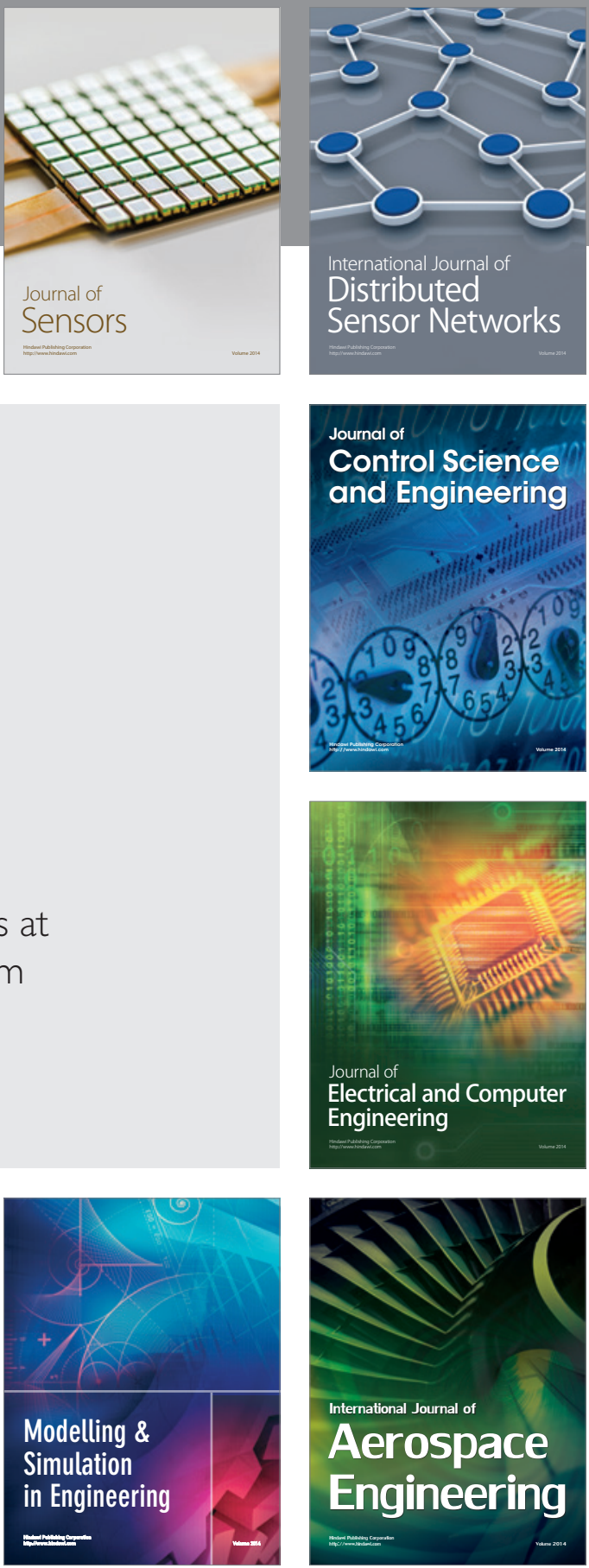

Journal of

Control Science

and Engineering
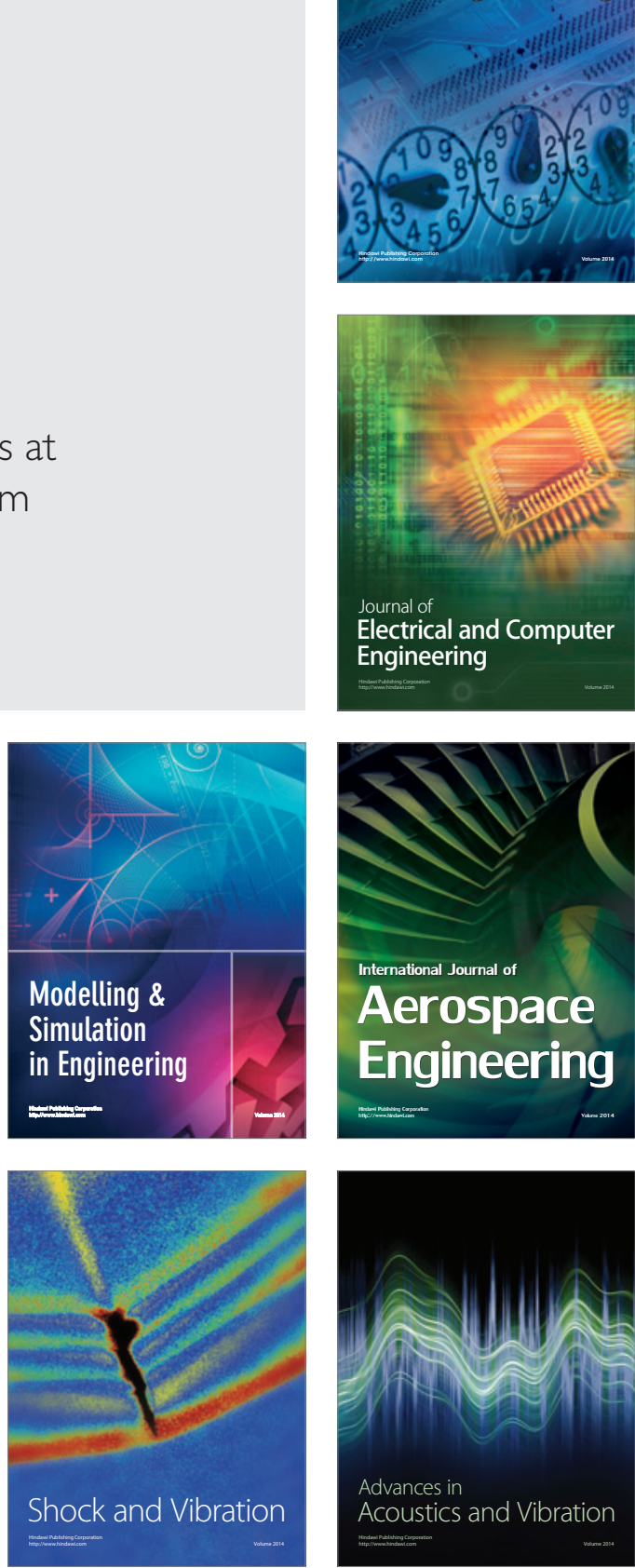